\title{
An interval Kalman filter-based fuzzy multi-sensor fusion approach for fault-tolerant heading estimation of an autonomous surface vehicle
}

\section{Motwani, A}

http://hdl.handle.net/10026.1/3636

\section{$10.1177 / 1475090215596180$}

Proceedings of the Institution of Mechanical Engineers, Part M: Journal of Engineering for the Maritime Environment

SAGE Publications

All content in PEARL is protected by copyright law. Author manuscripts are made available in accordance with publisher policies. Please cite only the published version using the details provided on the item record or document. In the absence of an open licence (e.g. Creative Commons), permissions for further reuse of content should be sought from the publisher or author. 


\title{
An interval Kalman filter-based fuzzy multi-sensor fusion approach for fault-tolerant heading estimation of an autonomous surface vehicle
}

Engineering for the Maritime Environment $1-17$

(c) IMechE 2015

Reprints and permissions: sagepub.co.uk/journalsPermissions.nav DOI: |0.||77/|4750902|5596|80 pim.sagepub.com @SAGE

\author{
Amit Motwani', Wenwen Liu', Sanjay Sharma', \\ Robert Sutton' and Richard Bucknall ${ }^{2}$
}

\begin{abstract}
This article presents a novel fuzzy-logic based multi-sensor data fusion algorithm for combining heading estimates from three separate weighted interval Kalman filters to construct a robust, fault-tolerant heading estimator for the navigation of the Springer autonomous surface vehicle. A single, low-cost gyroscopic unit and three independent compasses are used to acquire data onboard the vehicle. The gyroscope data, prone to sporadic bias drifts, are fused individually with readings from each of the compasses via a weighted interval Kalman filter. Unlike the standard Kalman filter, the weighted interval Kalman filter is able to provide a robust heading estimate even when subject to such gyroscope bias drifts. The three ensuing weighted interval Kalman filter estimates of the vehicle's heading are then fused via a fuzzy logic algorithm designed to provide an accurate heading estimate even when two of the three compasses develop a fault at any time. Simulations and real-time trials demonstrate the effectiveness of the proposed method.
\end{abstract}

\section{Keywords}

Multi-sensor data fusion, interval Kalman filtering, fuzzy logic, autonomous surface vehicle

Date received: I5 August 2014; accepted: II June 2015

\section{Introduction and background}

The roles envisaged for autonomous surface vehicles (ASVs) are rapidly expanding, with proposed applications not just in the military domain but also for civilian, commercial, industrial and research purposes. ${ }^{1}$ Therefore, there is a growing interest in developing autonomous navigation systems for these types of vehicles that are both reliable and low cost.

The operation of the Springer ASV is based on the integration of navigation, guidance and autopilot subsystems, the reliability of the whole being dependent upon the performance of each individual subsystem. A block diagram of this architecture is shown in Figure 1. The aim is to automatically steer the vehicle along a trajectory specified by a mission plan, using the guidance system that generates the desired or reference heading of the vehicle for each time step. The navigation system uses sensors to determine the vehicle's actual heading angle, and the autopilot generates the necessary thruster demands to adjust the vehicle's heading to maintain its course.
Although the focus of this article is on the heading estimation for the navigation subsystem, the other subsystems are utilised for holistic simulation purposes and in real-time trials, hence are also briefly described in this section, along with the background information on the Springer ASV. However, a mode detailed analysis on the estimation of the vehicle using Kalman filtering ensues and serves as the basis for the techniques developed in the following sections.

\footnotetext{
'Marine and Industrial Dynamic Analysis (MIDAS) Research Group, School of Marine Science and Engineering, Plymouth University, Plymouth, UK

${ }^{2}$ Marine Research Group, Department of Mechanical Engineering, University College London, London, UK
}

\section{Corresponding author:}

Amit Motwani, Marine and Industrial Dynamic Analysis (MIDAS)

Research Group, School of Marine Science and Engineering, Plymouth

University, Drake Circus, Plymouth, Devon PL4 8AA, UK.

Email: amit.motwani@plymouth.ac.uk 


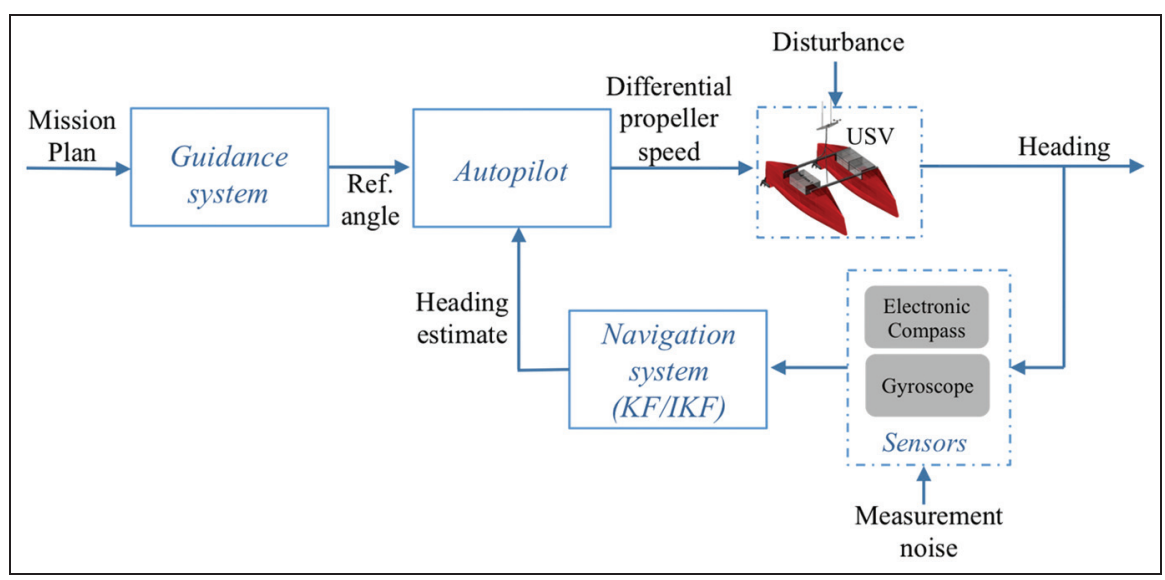

Figure I. Navigation, guidance and control block diagram.

\section{Objectives and outline}

The Kalman filter (KF) provides statistically optimal heading estimates, but it does so only if certain modelling hypotheses are met. It would become unreliable if the predicted behaviour of any component was to change, such as the development of a sensor fault. Multi-sensor data fusion (MSDF) techniques are software approaches that combine information from multiple sensors to improve reliability and accuracy. Sensor fusion based on KF technology has been widely used in navigation systems..$^{2-5}$ If redundant information is available, the system can be made fault tolerant. While numerous techniques exist, one approach, known as decentralised Kalman filtering, is based on fusing estimates from various local KFs. ${ }^{6}$ The method described here is one such technique, but its novelty lies in that it has been designed with the intent of being able to fuse individual weighted interval Kalman filter (wIKF) estimates as well.

The wIKF is a recent development in robust filtering techniques that extends the applicability of the KF to systems with finite modelling uncertainty, relaxing the restrictive hypotheses of the KF. The wIKF and its application to the heading estimation of Springer are briefly summarised in section 'Robustness and interval Kalman filtering', with the advantage of employing it over the KF when unforeseen gyroscope biases may develop also being explained.

The MSDF algorithm previously developed is then applied to the fusion of wIKF estimates, providing a system that is both fault tolerant and robust to modelling uncertainty. The results of applying the method in real-time trials with the Springer ASV are then presented, followed by a concluding section.

\section{Springer ASV}

Springer (Figure 2) is a medium waterplane twin hull battery-powered vessel designed as a test bed for developing autonomous capabilities. The 4-m-long and 2.3$\mathrm{m}$-wide vehicle is propelled by two $24-\mathrm{V}, 74-\mathrm{lbs}$ electric

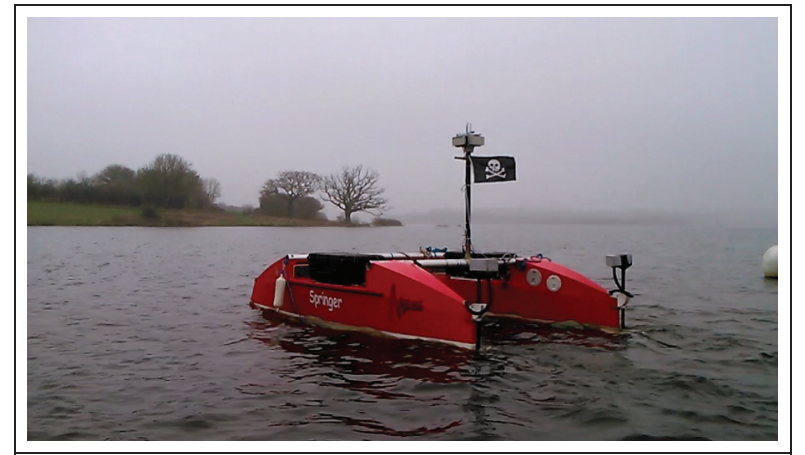

Figure 2. Springer ASV during trials at Roadford Lake, Devon, UK.

ASV: autonomous surface vehicle.

Minn Kota Riptide ${ }^{\circledR}$ transom-mounted saltwater trolling motors. Particular focus is given to steering control, achieved by controlling the difference in propeller revolution rates.

The vehicle is equipped with onboard computers contained in watertight Pelican $^{\text {TM }}$ Cases on each hull. The computers are connected to a motor driver housed within one of the hulls allowing them to command the motors, and a WiFi router atop a mast enabling them to be remotely accessed for the purposes of mission supervision and configuration. The mast also houses Global Positioning System (GPS) units which transmit localisation data to the computers. For heading estimation, one of the Pelican Cases houses a suite of electronic compasses and a gyroscopic unit, details of which are described next, whereas further details of other hardware can be found in a previous publication. ${ }^{7}$

\section{Springer navigational suite}

The navigational suite of Springer includes a low-cost micro-electromechanical systems (MEMS) gyroscope unit and three digital magnetic compasses, the main characteristics of which are summarised in Table 1.

The sensorial redundancy may appear wasteful, but in practice, sensor failure is a common occurrence, 
Table I. Springer sensor suite for heading determination.

\begin{tabular}{|c|c|c|}
\hline Unit & Technology & Measurement noise ${ }^{a}$ \\
\hline TinkerKit gyroscope & $\begin{array}{l}\text { Two-axis MEMS technology gyro, based on the LPR5I50AL from } \\
\text { ST Microelectronics }\end{array}$ & $0.05^{\circ} \mathrm{s}^{-1} \mathrm{RMS}$ \\
\hline TCM2 compass & $\begin{array}{l}\text { Magneto-inductive effect three-axis magnetometer with two-axis } \\
\text { inclinometer for tilt compensation }\end{array}$ & $I^{\circ} \mathrm{RMS}$ \\
\hline HMR3000 compass & $\begin{array}{l}\text { Magneto-resistive sensors in three orthogonal directions, with } \\
\text { fluidic tilt sensor for tilt-compensated heading }{ }^{10}\end{array}$ & $0.5^{\circ} \mathrm{RMS}$ \\
\hline $\mathrm{KVH} \mathrm{CIO0} \mathrm{compass}$ & Flux-gate compass with iron magnetic compensation ${ }^{\prime \prime}$ & $0.5^{\circ} \mathrm{RMS}$ \\
\hline
\end{tabular}

MEMS: micro-electromechanical systems; RMS: root mean square.

${ }^{a}$ Approximate values obtained from testing the devices in the trials environment. The compasses were sampled at I Hz, corresponding to the overall sampling time used in the vehicle's control loop. The gyroscope was sampled at the faster rate of $5 \mathrm{~Hz}$ and the RMS noise value is the result of smoothing the acquired signal over a period of I s.

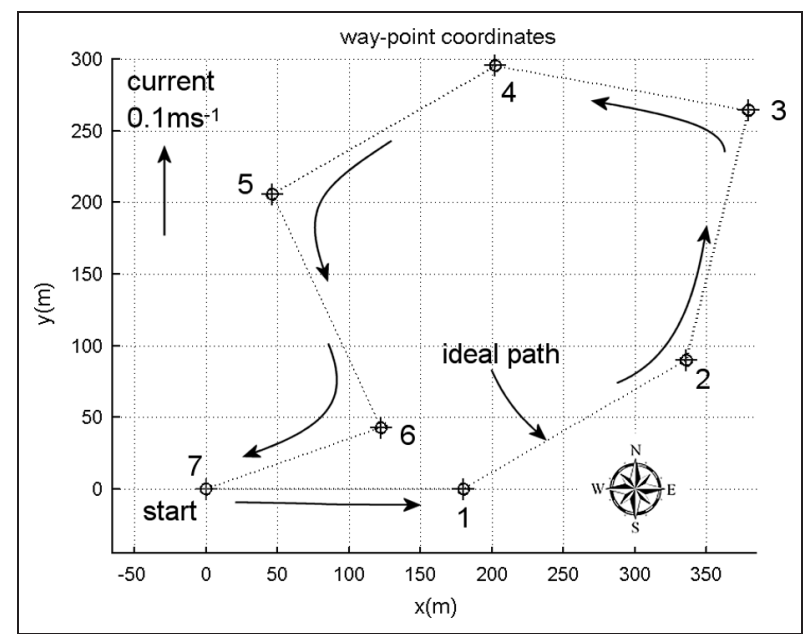

Figure 3. Way-point tracking mission.

especially when low-cost hardware is involved. By way of example, during some recent trials undertaken with the ASV, a sporadic communications error between one of the compasses and the main onboard computer impeded the transmission of data between these. In this case, it sufficed to manually switch to another compass, but during an autonomous mission, such a luxury would not exist and the occurrence of a hardware failure during the course of a mission would most likely result in its forced abortion.

\section{Autonomous way-point tracking}

Consider the tracking mission depicted in Figure 3. The mission plan is a series of specified way-points with known coordinates that the vehicle must pass through, for which at each time step, the guidance system generates a reference heading angle according to

$$
r(k)=\arctan \left(\frac{y_{d}(k)-y^{\mathrm{ASV}}(k)}{x_{d}(k)-x^{\mathrm{ASV}}(k)}\right)
$$

where $x^{\mathrm{ASV}}$ and $y^{\mathrm{ASV}}$ represent the current locations of the vehicle, assumed to be known at each sampling time via a GPS receiver, and $x_{d}$ and $y_{d}$ the target coordinates or coordinates of the next way-point. The autopilot then assesses the difference between this desired reference heading and the actual heading of the vehicle in order to determine the control action or differential motor speed that must be applied to keep the vehicle on course. Although more sophisticated autopilots have been proposed for ASV steering, ${ }^{12}$ for this study, a simple state-feedback autopilot is implemented based on a linear state-space model of the vehicle's steering dynamics obtained through system identification techniques. The model provides the vehicle turning rate in terms of the applied difference in motor revolution rates for a given vehicle speed, in this case for a fixed value of $1.5 \mathrm{~m} \mathrm{~s}^{-1}$, and is also used to simulate the vehicle's motion. Details of the vehicle model and the autopilot are given in Appendix 1.

It should be mentioned that in the simulations carried out in this article, a disturbance was added whereby the water surface was assumed to be moving with a current of $0.1 \mathrm{~m} \mathrm{~s}^{-1}$ in a northerly direction, transporting the vehicle along this direction without affecting its heading. The purpose of this additional disturbance is related to autopilot capability studies and does not directly affect the study herein.

\section{Kalman filtering for heading estimation}

Let $\Omega_{i}$ represent the actual turning rate of the vehicle and $\Omega_{0}$ the gyroscope reading, given by

$$
\Omega_{0}=\Omega_{i}+\omega
$$

where $\omega$ is the random noise associated with the measurement. The heading of the vehicle can be obtained by discrete integration of the turning rate, that is

$$
\theta(k+1)=\theta(k)+T_{s} \times \Omega_{i}(k)
$$

where $T_{s}$ is the sampling time and $k$ is the number of time steps. In terms of the gyroscope reading, this is equivalent to

$$
\theta(k+1)=\theta(k)+T_{s} \times\left[\Omega_{0}(k)-\omega(k)\right]
$$


Given an initial heading estimate $\hat{\theta}(0)$, the subsequent headings can, of course, be estimated from the successive gyroscope measurements alone

$$
\hat{\theta}(k)=\hat{\theta}(0)+T_{s} \sum_{i=0}^{k-1} \Omega_{0}(i)
$$

Assuming that the time-sampled noise sequence $\{\omega(k)\}$ is white noise and normally distributed with variance $q$ and $\mathrm{E} \hat{\theta}(0)=\theta(0)$, then the estimate is unbiased in the sense that

$$
\begin{aligned}
\mathrm{E} \hat{\theta}(k) & =\mathrm{E} \hat{\theta}(0)+T_{s} \sum_{i=0}^{k-1} \mathrm{E} \Omega_{0}(i) \\
& =\theta(0)+T_{s} \sum_{i=0}^{k-1} \Omega_{i}(i)=\theta(k)
\end{aligned}
$$

However, its variance grows linearly with time

$$
\begin{aligned}
\operatorname{var} \hat{\theta}(k) & =\operatorname{var} \hat{\theta}(0)+T_{s}^{2} \operatorname{var} \sum_{i=0}^{k-1} \Omega_{0}(i) \\
& =\operatorname{var} \hat{\theta}(0)+T_{s}^{2} \sum_{i=0}^{k-1} \operatorname{var} \omega(i)=\operatorname{var} \hat{\theta}(0) \\
& +k T_{s}^{2} q
\end{aligned}
$$

that is, with roughly $68 \%$ probability the root mean square (RMS) error of the estimate will lie between $\pm \sqrt{k} T_{s} q^{1 / 2}$ after $k$ time steps.

Not only does the variance of the estimation error increase with time but also the resulting error sequence will no longer be white noise, but rather, what is known as a random walk, causing the estimate to drift from the true value, a phenomenon which in this case is commonly referred to as gyro integration drift.

Figure 4(a) depicts, for an initial 100-time step simulation of the way-point tracking mission, the actual turning rate of the vehicle, $\Omega_{i}(k)$, as compared to simulated gyroscope readings of the same, $\Omega_{0}(k)$, for which the noise $\omega(k)$ is generated pseudo randomly according to a normal distribution with zero mean and variance $q=0.05^{2}\left({ }^{\circ} \mathrm{s}^{-1}\right)^{2}$ (Figure 4(c)). The actual vehicle heading $\left(\theta(k)=\theta(0)+T_{s} \sum_{i=0}^{k-1} \Omega_{i}(i) ; \theta(0)=0\right)$ is shown in Figure 4(b) along with the heading obtained from integration of the gyroscope readings (equation (5), where $\hat{\theta}(0)=0$ ), and the difference between these two, $\hat{\theta}(k)-\theta(k)=T_{s} \sum_{i=0}^{k-1} \omega(i)$, is plotted in Figure 4(d). The plot shown in Figure 4(d) is characteristic of a random walk, and the accumulated heading prediction error is around $0.8^{\circ}$, which is a likely value, given $\sqrt{100} \times 1 \times 0.05=0.5^{\circ}$. The system operates at a sampling time $T_{s}=1 \mathrm{~s}$.

Figure 4(b) also shows simulated compass readings, $z_{\theta}(k)$, generated as pseudo random values drawn from the normal distribution with mean equal to the actual vehicle heading at each time step, $\theta(k)$, and variance $r=1^{\circ 2}$

$$
z_{\theta}(k)=\theta(k)+\nu(k),\{\nu(k)\} \text { white noise } \sim N(0, r=1)
$$

Unlike the gyroscope estimate, compass readings do not suffer from integration drift; however, the measurement noise is typically larger than that of an inertial sensor, and smoothing of the signal due to integration is not present. Hence, direct measurement of the heading produces a relatively noisy signal. In order to exploit the precision of the gyroscope for short-term estimation together with the long-term accuracy of the compass, the KF has long been the data fusion algorithm of choice.

Defining the state variable $x(k)$ as the heading angle at each time step, equation (4) can be viewed as the state equation for predicting the next state given the current state and known input

$$
x(k+1)=x(k)+T_{s} u(k)-T_{s} \omega(k)
$$

where the input $u(k)$ is the gyroscope reading, $\Omega_{0}(k)$. The measurement equation is given by the compass measurement model

$$
z_{\theta}(k)=x(k)+\nu(k)
$$

Equations (9) and (10) are of the standard form

$$
\begin{aligned}
& \boldsymbol{x}(k+1)=\mathbf{A} \boldsymbol{x}(k)+\mathbf{B} \boldsymbol{u}(k)+\boldsymbol{\omega}(k) \\
& \boldsymbol{z}(k)=\mathbf{C} \boldsymbol{x}(k)+\boldsymbol{\nu}(k) \\
& \boldsymbol{x}(k), \boldsymbol{\omega}(k) \in \Re^{n}, \boldsymbol{u}(k) \in \Re^{m}, \boldsymbol{z}(k), \boldsymbol{\nu}(k) \in \Re^{l}, \\
& \mathbf{A} \in \Re^{n \times n}, \mathbf{B} \in \Re^{n \times m} \text { and } \mathbf{C} \in \Re^{l \times n}
\end{aligned}
$$

with $n=m=l=1$ and $\omega(k)$ and $\nu(k)$ mutually independent white noise sequences, with zero-mean normal distributions with known covariances $q$ and $r$, respectively, for which, given an initial estimate of the state, $\hat{x}(0)$, and of the state estimate error covariance, $P(0)=\mathrm{E}[x(0)-\hat{x}(0)]^{2}$, the KF (Appendix 2) can be applied. Under these assumptions, the KF provides statistically optimal estimates of the system state. ${ }^{13}$

The sequence of KF heading estimates for the aforementioned vehicle simulation, $\hat{\theta}_{K F}(k)$, given $\hat{\theta}_{K F}(0)=0$ and $P(0)=0$, is shown in Figure 4(b). A quantitative assessment of each estimation method is provided in Table 2. It shows that while the gyroscope predictions are more accurate than the compass measurements for short-term prediction, in the long term, the gyroscopebased prediction RMS error increases indefinitely with time. However, the KF estimate, which fuses the gyroscope prediction with compass measurement, is more accurate than either of these taken individually (note that this is true at least statistically, even though what is shown here is a particular realisation of said statistics). Moreover, the detectability of $\mathbf{A}$ and $\mathbf{C}$ guarantees that the error covariance of the KF remains bounded, and in this case, it is trivial to verify as the system is scalar. ${ }^{14}$ Note that stability of the filter guarantees that the estimation error will be bounded, regardless of whether the plant being regulated is stable or not. 


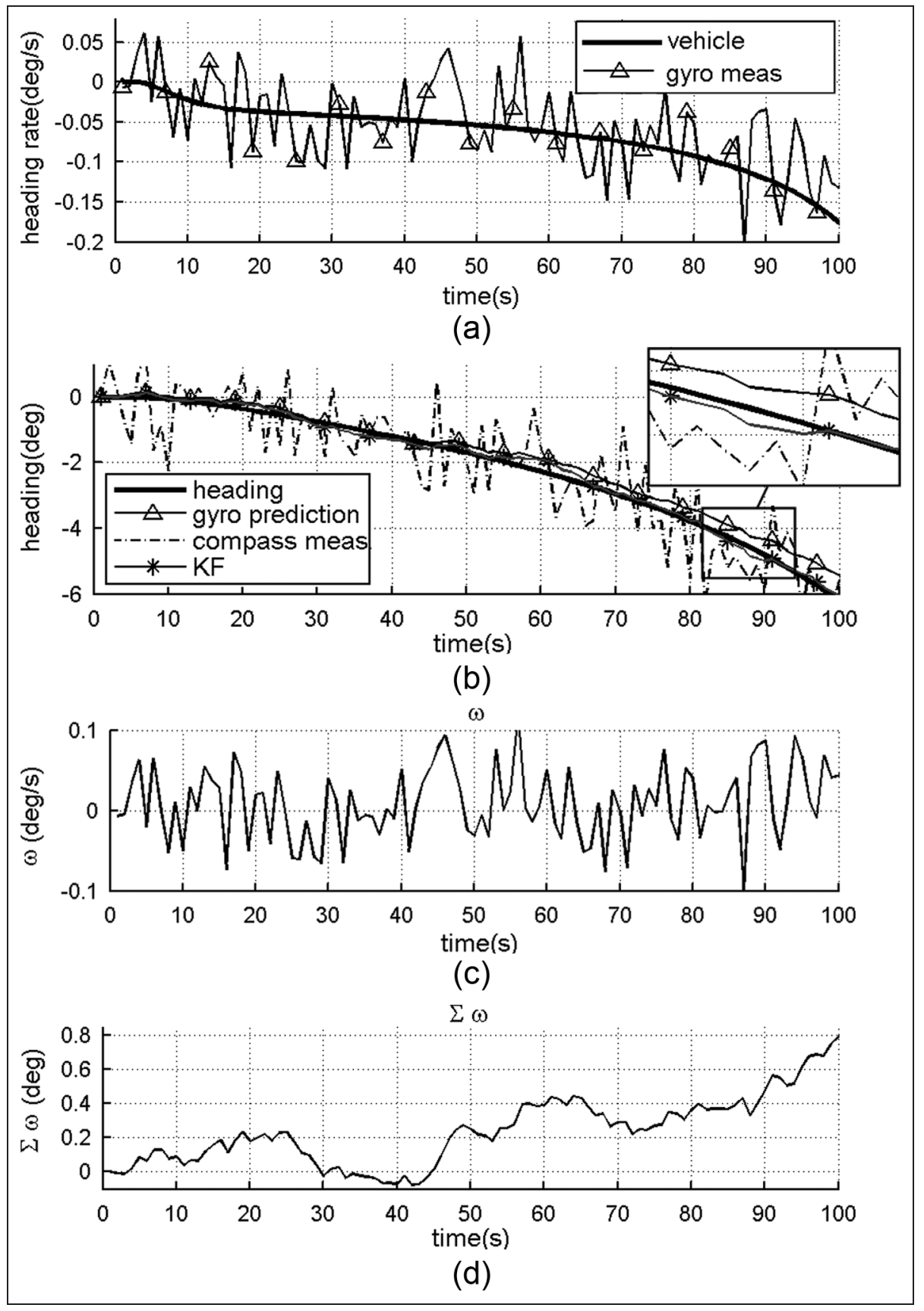

Figure 4. Initial 100-time step simulation of the tracking mission: (a) actual turning rate of the vehicle and (noisy) gyroscope measurement; (b) actual heading of the vehicle, predicted heading based on dead reckoning from gyroscope readings, (noisy) compass measurements and KF heading estimate; (c) gyroscope noise; and (d) integrated gyroscope noise (random walk). KF: Kalman filter.

Table 2. Comparison of heading estimate errors.

\begin{tabular}{lll}
\hline Method for heading estimation & Heading RMS error from $k=I$ to $N\left({ }^{\circ}\right)$ \\
\hline Gyroscope reading integration (dead reckoning) $e_{\text {gyro }}=\left\{N^{-1} \sum_{k=1}^{N}[\theta(k)-\hat{\theta}(k)]^{2}\right\}^{1 / 2}$ & 0.312 & $N=200$ \\
Compass measurements $e_{z}=\left\{N^{-1} \sum_{k=1}^{N}\left[\theta(k)-z_{\theta}(k)\right]^{2}\right\}^{1 / 2}$ & 0.903 \\
Kalman filter estimate $e_{K F}=\left\{N^{-1} \sum_{k=1}^{N}\left[\theta(k)-\hat{\theta}_{K F}(k)\right]^{2}\right\}^{1 / 2}$ & 0.013 & 0.965
\end{tabular}

RMS: root mean square. 


\section{Fuzzy data fusion algorithm for Kalman estimates}

The Springer ASV is, as detailed in section 'Introduction and background', equipped with a gyroscope and three magnetic compasses. A KF can be built to fuse data between the gyroscope and each individual compass, as explained previously, resulting in three distinct KFs that are identical in their predictive models (equation (9)) but with different compass measurement noise covariances. However, if a compass was to fail, the corresponding $\mathrm{KF}$ performance would degrade significantly. Hence, there should exist some mechanism by which a faulty KF estimate should automatically be rejected in the vehicle's navigation system.

The fuzzy data fusion algorithm proposed here assigns a weight to each of the three KF estimates, so that the fused state estimate may be computed as

$$
\hat{\theta}_{f K F}(k)=\sum_{i=1}^{3} w_{i}(k) \quad \hat{\theta}_{K F_{i}}(k)
$$

Being a linear combination of the individual KF estimates, the stability of the fused estimate follows from that of each individual filter. The weighting decision is based on assessing the innovation sequence of each KF. A KF innovation sequence is defined as the difference between the measurement of the output and the predicted value

$$
\{\boldsymbol{i n n}(k)\}=\{\boldsymbol{z}(k)-\boldsymbol{C} \hat{\boldsymbol{x}}(k \mid k-1)\}
$$

which in this case corresponds simply to the difference between the compass measurement at time $k$ and the predicted heading given the previous heading estimate and gyroscope reading. It is well established that in an ideal scenario, the innovation sequence should be composed of zero-mean white noise. ${ }^{2,15}$ Thus, a KF's innovation sequence could be monitored to detect a failure in the KF which may then be used to penalise its contribution in equation (13).

In order to monitor the innovation, which in general is a random process, hence its values when considered individually are meaningless, a simple moving average (SMA) is computed

$$
\operatorname{SMA}(k)=m^{-1} \sum_{i=k-m+1}^{k} \operatorname{inn}(i)
$$

$m$ being the number of samples considered in the moving average. Since the SMA is, in the ideal case, a sum of zero-mean independent random variables, it is in itself a zero-mean random variable, tending to be normally distributed by the Central Limit Theorem. However, its variance is $m$ times smaller than that of the innovation random variable. Thus, sporadic high values of the SMA occur with less likelihood, and sustained occurrences of these will be indicative of an innovation that is no longer white noise. Hence, it is

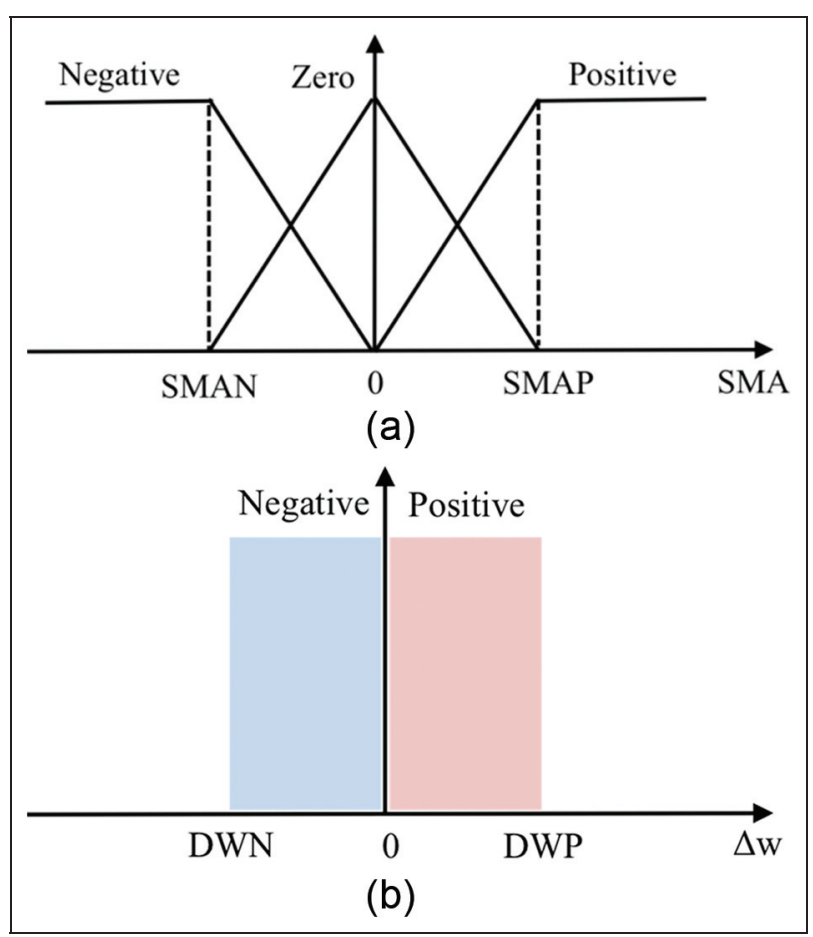

Figure 5. (a) Input and (b) output membership functions.

this value that is monitored to indicate a compass fault in the KF estimate.

Based on this idea, the following rules would adequately modify a KF's contribution to the fused estimate: if the SMA is somewhat larger or smaller than zero, decrease the weight of the corresponding KF; else if it is zero, increase its weight. In order to quantify these statements, consider the following membership functions, shown graphically in Figure 5, in which SMAN, SMAP, DWN and DWP are some threshold values:

\section{- Input membership functions}

Negative function :

$$
\mu_{N}^{i}=\left\{\begin{array}{cc}
1 & \text { if } \mathrm{SMA}<\mathrm{SMAN} \\
\text { SMA } / \mathrm{SMAN} & \text { if } \mathrm{SMAN} \leqslant \mathrm{SMA}<0 \\
0 & \text { if } \mathrm{SMA} \geqslant 0
\end{array}\right.
$$

Zero function :

$$
\mu_{Z}^{i}=\left\{\begin{array}{l}
1-\mathrm{SMA} / \mathrm{SMAN} \quad \text { if } \mathrm{SMAN} \leqslant \mathrm{SMA}<0 \\
1-\mathrm{SMA} / \mathrm{SMAP} \quad \text { if } 0 \leqslant \mathrm{SMA} \leqslant \mathrm{SMAP}
\end{array}\right.
$$

Positive function :

$$
\mu_{P}^{i}=\left\{\begin{array}{cc}
0 & \text { if } \mathrm{SMA}<0 \\
\text { SMA } / \mathrm{SMAP} & \text { if } 0 \leqslant \mathrm{SMA}<\mathrm{SMAP} \\
1 & \text { if } \mathrm{SMA} \geqslant \mathrm{SMAP}
\end{array}\right.
$$


- Output membership functions

Negative function $: \mu_{N}^{o}=\left\{\begin{array}{cc}1 & \text { if } \mathrm{DWN} \leqslant \mathrm{SMA}<0 \\ 0 & \text { otherwise }\end{array}\right.$

Positive function : $\mu_{P}^{o}=\left\{\begin{array}{cc}1 & \text { if } 0 \leqslant \mathrm{SMA}<\mathrm{DWP} \\ 0 & \text { otherwise }\end{array}\right.$

In order to avoid brusque transitions in the combined estimate, the output to the fuzzy logic inference system is chosen to be a change in the weight of the filter, $\Delta w$, rather than the weight itself (Figure 5(b)).

Based upon the aforementioned membership functions, the following fuzzy rules are established:

- Rule 1: If SMA is negative, then $\Delta w$ is negative;

- Rule 2: If SMA is zero, then $\Delta w$ is positive;

- Rule 3: If SMA is positive, then $\Delta w$ is negative.

Then, at each sampling time $k$, depending upon the value of the SMA, $\Delta w$ is computed as follows:

- Case 1: SMA < SMAN-Rule 1 applies and $\Delta w$ is given by the horizontal projection of the centroid of the negative output membership function, that is, $\Delta w=\mathrm{DWN} / 2$.

- Case 2: SMAN < SMA $\leqslant 0$ - Both Rule 1 and Rule 2 apply. Let $\mu_{N}^{i}$ represent the degree of membership of the input to the negative input membership function (Rule 1), and $\mu_{Z}^{i}$ its degree of membership to the zero input membership function (Rule 2). Then, $\Delta w$ is computed as the horizontal projection of the centroid of the area comprising the portions of the negative and positive output membership functions below the values $\mu_{N}^{i}$ and $\mu_{Z}^{i}$, respectively (Figure 6)

$$
\Delta w=\frac{-\frac{1}{2} \mathrm{DWN}^{2} \times \mu_{N}^{i}+\frac{1}{2} \mathrm{DWP}^{2} \times \mu_{Z}^{i}}{-\mathrm{DWN} \times \mu_{N}^{i}+\mathrm{DWP} \times \mu_{Z}^{i}}
$$

- Case 3: $0<$ SMA < SMAP - Both Rule 2 and Rule 3 apply. Let $\mu_{Z}^{i}$ represent the degree of membership of the input to the zero input membership function (Rule 2), and $\mu_{P}^{i}$ its degree of membership to the positive input membership function (Rule 3). Then, $\Delta w$ is computed as the horizontal projection of the centroid of the area comprising the portions of the positive and negative output membership functions below the values $\mu_{Z}^{i}$ and $\mu_{P}^{i}$, respectively

$$
\Delta w=\frac{-\frac{1}{2} \mathrm{DWN}^{2} \times \mu_{P}^{i}+\frac{1}{2} \mathrm{DWP}^{2} \times \mu_{Z}^{i}}{-\mathrm{DWN} \times \mu_{P}^{i}+\mathrm{DWP} \times \mu_{Z}^{i}}
$$

- Case 4: SMAP $\leqslant$ SMA -Rule 3 solely applies, and $\Delta w$ is given by the horizontal projection of the centroid of the negative output membership function, that is, $\Delta w=\mathrm{DWN} / 2$.

Once $\Delta w$ has been calculated at time step $k$ for each $\mathrm{KF}\left(\Delta w_{i}(k), i=1,2,3\right)$, these values can be normalised, so that their sum equals zero to ensure that the sum of the weights themselves remains equal to 1 (the weights are initialised equally at $1 / 3$ for $k=0$ )

$$
\begin{aligned}
& \Delta w_{i}^{*}(k)=\Delta w_{i}(k)-\alpha, \quad i=1,2,3, \\
& \text { with } \alpha \text { such that } \sum_{i=1}^{3}\left(\Delta w_{i}-\alpha\right)=0, \text { that is, } \\
& \alpha=\frac{1}{3} \sum_{i=1}^{3} \Delta w_{i}
\end{aligned}
$$

resulting in the updated weights of each filter given by

$$
w_{i}(k):=w_{i}(k-1)+\Delta w_{i}^{*}(k), \quad i=1,2,3
$$

However, direct application of equation (24) might yield updated weights outside of the interval $[0,1]$. To restrict the values of the weights to this interval, the following redistribution procedure is performed. Instead of directly updating all the weights according to equation (24), these are tentatively updated in some auxiliary variables

$$
w_{i}^{*}:=w_{i}(k-1)+\Delta w_{i}^{*}(k), \quad i=1,2,3
$$

The following three possibilities exist:

- If all $w_{i}^{*}$ are between 0 and 1 (inclusive), then these are taken directly as the updated weights $w_{i}(k)$ (equation (24)).

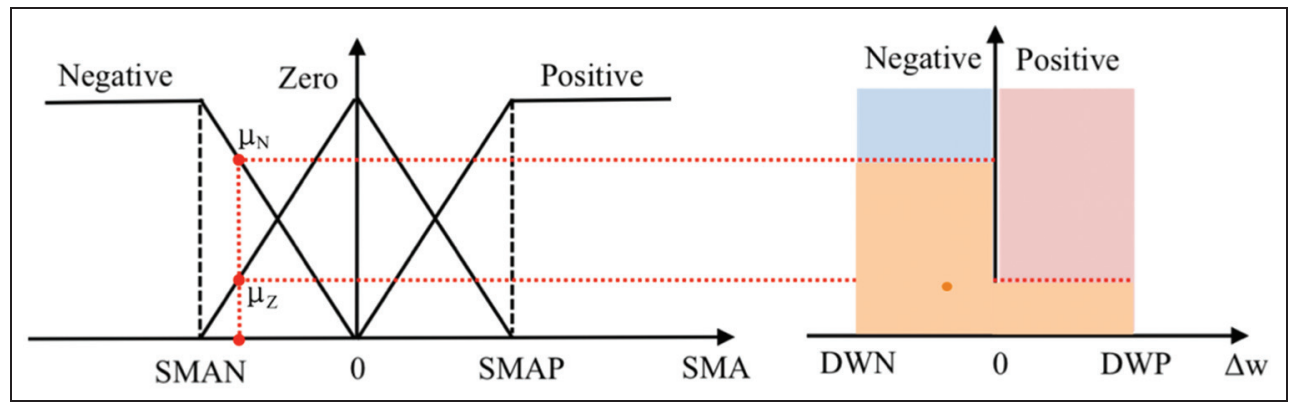

Figure 6. Calculation of the output $\Delta w$ for Case 2 (SMAN $<$ SMA $\leqslant 0$ ). 


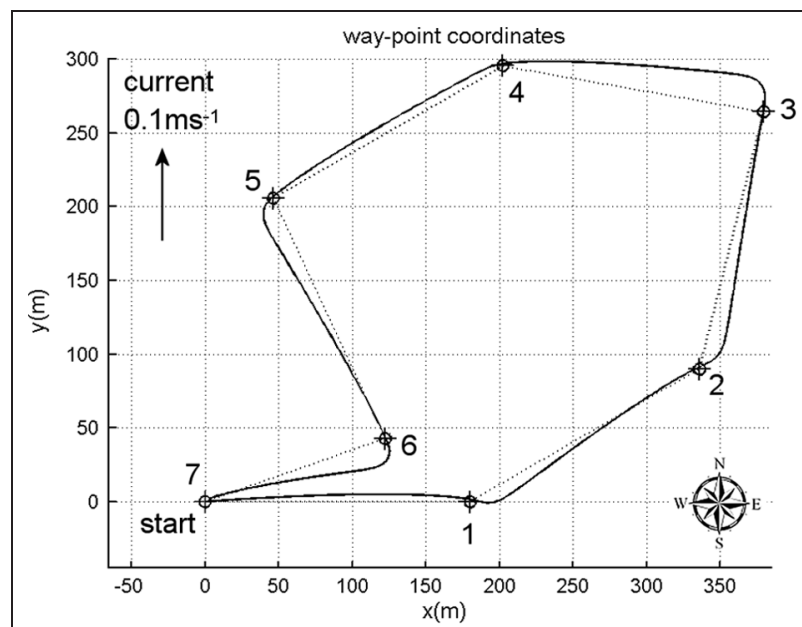

(a)

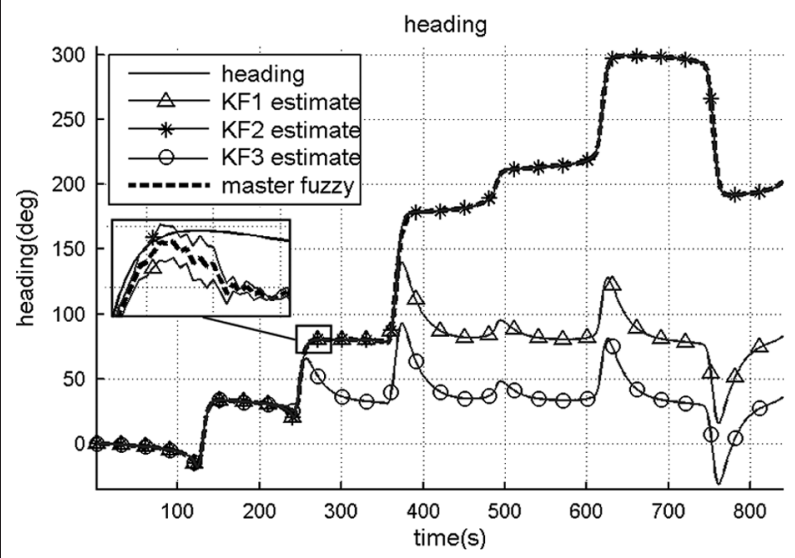

(b)

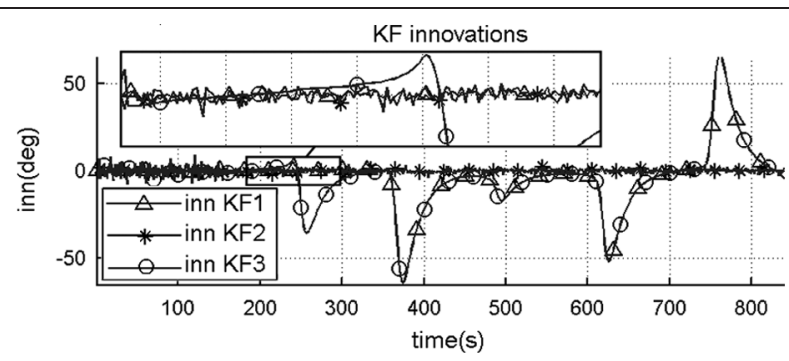

(c)

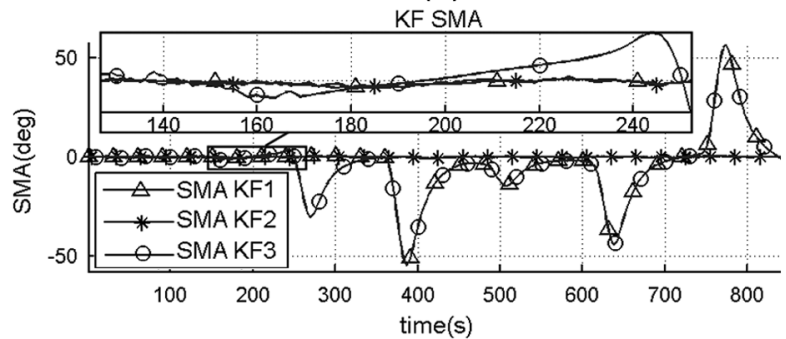

(d)

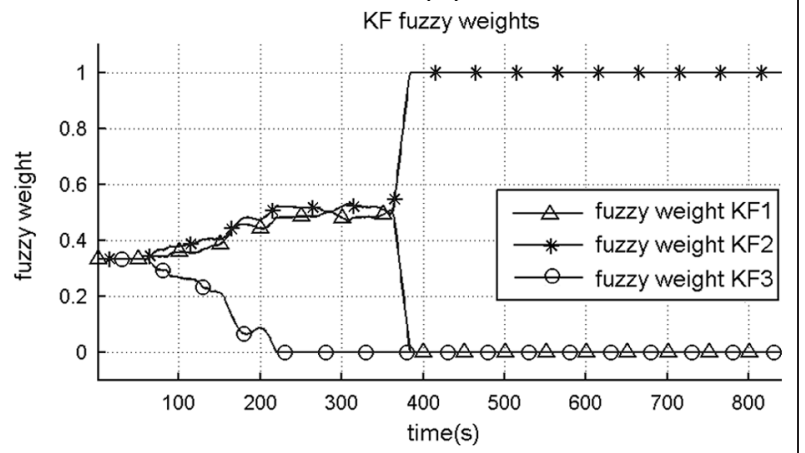

(e)

Figure 7. Simulation of way-point tracking mission: (a) actual path taken by the vehicle; (b) actual heading, KF heading estimates and fuzzy logic-fused KF estimate; (c) innovations sequence of each KF; (d) SMA of each KF innovation; and (d) fuzzy weights assigned to each KF.

KF: Kalman filter; SMA: simple moving average.

- If (only) one of the $w_{i}^{*}$ is less than zero, for example, $\quad w_{j}^{*}<0, \quad$ then $\Delta w_{j}^{* *}$ is defined as $\Delta w_{j}^{* *}:=-w_{j}(k-1)$, that is, the part of $\Delta w_{j}(k)$ that is actually used to make the corresponding updated weight equal to zero. Then, the remaining two weight increments are normalised again: $\Delta w_{i}^{* *}(k):=\Delta w_{i}^{*}(k)-\alpha, i=1,2,3$ and $i \neq j$, with $\alpha$ such that $\Delta w_{j}^{* *}(k)+\sum_{\substack{i=1 \\ i \neq j}}^{3}\left(\Delta w_{i}^{*}-\alpha\right)=0$, whereby $\alpha=(1 / 2)\left[\Delta w_{j}^{* *}(k)+\sum_{\substack{i=1 \\ i \neq j}}^{3} \Delta w_{i}^{*}\right]$. The new prospective weights are then given by $w_{i}^{* *}=w_{i}(k-1)+$ $\Delta w_{i}^{* *}(k), i=1,2,3$, where in particular $w_{j}^{* *}=$ $w_{j}(k-1)+\Delta w_{j}^{* *}(k)=0$. If none of the resulting $w_{i}^{* *}$ are negative, then these are the updated weights $w_{i}(k)$; however, if one of them is negative, for example, $w_{l}^{* *}<0$, then the updated weights are $w_{j}(k)=0, \quad w_{l}(k):=0$ and $w_{i}(k):=1, i \in\{1,2,3\}$ and $i \neq j, l$.

- If two of the $w_{i}^{*}$ obtained using equation (25) are negative, for example, $w_{j}^{*}<0$ and $w_{l}^{*}<0$, this implies that the third weight, $w_{i}^{*}, i \in\{1,2,3\}$ and $i \neq j, l$, will be larger than one, since the sum of the three is always equal to unity. Therefore, it suffices to take $w_{j}(k):=0, w_{l}(k):=0$ and $w_{i}(k):=1$.

This scheme allows for weights that at some point devolve to a zero value, signifying complete rejection of the corresponding $\mathrm{KF}$, to start recovering if and when they are subsequently prescribed positive weight increments. A similar scheme without recovery is easily implemented by flagging down a KF that is assigned a zero weight at any given time, thenceforth permanently assigning it a zero weight and carrying out the weight redistribution process among the remaining filters.

The fuzzy logic assignment of weight increments and ensuing normalisation or redistribution occurs only after an initial $m$ time steps, required to compute the SMA of the innovation, have elapsed, prior to which the weights are maintained equally at $1 / 3$.

Figure 7 shows a complete simulation of the vehicle way-point tracking mission, assuming the autopilot has 
Table 3. KF characteristics.

\begin{tabular}{lll}
\hline & Gyroscope noise model & Compass noise model \\
\hline $\mathrm{KF}_{1}$ & $q=0.05^{2}\left({ }^{\circ} \mathrm{s}^{-1}\right)^{2}$ & $r_{1}=0.25^{\circ 2}$ \\
$\mathrm{KF}_{2}$ & & $r_{2}=1^{\circ 2}$ \\
$\mathrm{KF}_{3}$ & & $r_{3}=9^{\circ 2}$ \\
\hline
\end{tabular}

KF: Kalman filter.

Table 4. Parameter values for fusion algorithm.

\begin{tabular}{ll}
\hline Parameter & Value \\
\hline SMA length & 20 \\
SMAN & -5 \\
SMAP & 5 \\
DWN & -0.05 \\
DWP & 0.05
\end{tabular}

SMA: simple moving average.

access to the system state vector (Appendix 1). Although not important to this study, for completeness, the actual path followed by the vehicle is shown in Figure 7(a). The vehicle steering model allows computation of the heading from the autopilot signal, and gyroscope readings and compass measurements are obtained as described in section 'Introduction and background'. Three different compasses are simulated, with noise processes $\nu_{i} \sim N\left(0, r_{i}\right)$, along with their respective KFs (fusion of gyroscope with each compass individually; Table 3), initialised with $\hat{\theta}_{K F_{i}}(0)=0$ and $P_{K F_{i}}(0)=0, i=1,2,3$.

In order to test the fault tolerance of the fused KF estimate, during the course of the simulation, two compasses are made to fail in such a way that their readings remain frozen at the last good value before failure. Concretely, the compass associated with $\mathrm{KF}_{3}$ freezes at $k=150$ and the one associated with $\mathrm{KF}_{1}$ at $k=350$. The actual vehicle heading at each time step and the three individual KF estimates are shown in Figure 7(b). It shows how, after a compass failure, the affected KF estimate tends to the respective frozen compass value when the gyroscope reading is small (i.e. during the straight line segments of the trajectory).

The fused KF estimate is also shown in Figure 7(b). In order to understand its progression, the innovations of each KF are shown in Figure 7(c), and their SMAs in Figure 7(d), based upon which the fuzzy logic system calculates the weight increments for each filter. The resulting weights are shown in Figure 7(d). The SMA length and fuzzy membership function threshold values, chosen heuristically, are given in Table 4. Recovery of weights was not permitted.

Table 5 summarises the RMS errors of the three KF estimates and the fused estimate. Note that the majority of the error of the fused estimate occurs due to the transient periods shortly after the compass failures, as the fusion weights need time to adjust.
Table 5. KF and fused KF estimate errors.

\begin{tabular}{ll}
\hline Method & Heading RMS error $\left(^{\circ}\right)$ \\
\hline $\mathrm{KF}_{1}$ & 112.75 \\
$\mathrm{KF}_{2}$ & 0.21 \\
$\mathrm{KF}_{3}$ & 147.28 \\
Fused estimate & 0.72 \\
\hline
\end{tabular}

KF: Kalman filter; RMS: root mean square.

\section{Robustness and interval Kalman filtering}

Navigation systems that rely on low-cost hardware such as MEMS gyroscopes and compasses for determining vehicle heading are subject to uncertainties inherent in such units, such as time- and temperaturedependent bias drifts. These uncertainties translate into inaccurate predictive or measurement models. The KF approach to fusing noisy sensor data that have been used so successfully with high-grade units, whose models are known with precision, is subject to serious degradation when applied to inaccurately modelled systems. However, it is the low cost and small size of MEMS sensors that enable their availability for widespread civilian applications. Hence, another arm of the Springer project also focuses on the development of a robust $\mathrm{KF}$ technique that yields accurate estimates even in the face of modelling uncertainty.

Consider a model such as the one given by equations (11) and (12), in which the elements of the matrices $\mathbf{A}, \mathbf{B}$ and $\mathbf{C}$ are not known precisely but known to lie within certain bounds. If these bounded uncertain elements are described as intervals, then the system can be described by an interval model rather than a pointvalued one (equations (26) and (27))

$$
\begin{aligned}
& \boldsymbol{x}(k+1)=\mathbf{A}^{\mathbf{I}} x(k)+\mathbf{B}^{\mathbf{I}} u(k)+\boldsymbol{\omega}(k) \\
& \boldsymbol{z}(k)=\mathbf{C}^{\mathbf{I}} x(k)+\boldsymbol{\nu}(k)
\end{aligned}
$$

in which $\mathbf{A}^{\mathbf{I}}, \mathbf{B}^{\mathbf{I}}$ and $\mathbf{C}^{\mathbf{I}}$ are interval-valued matrices, that is, their elements are made up of intervals of the form

$$
m_{i, j}^{I}=\left[m_{i, j}^{\min }, m_{i, j}^{\max }\right] ; \quad \text { with } m_{i, j}^{\min }, m_{i, j}^{\max } \in \Re
$$

where $\min$ and $\max$ refer to the minimum and maximum values of the interval, respectively. Based on this idea of interval model, and the appropriate concepts of interval mathematics, Chen et al. ${ }^{16}$ proposed the socalled interval Kalman filter (IKF) as a direct extension of the traditional KF for interval systems. However, the IKF provides state estimates in the form of intervals rather than point values, so that at each time step, there is an upper and lower bound to the estimate. Moreover, this interval estimate guarantees to contain every KF estimate that would be obtained based on any point-valued model contained in the interval model. Therefore, if the true system dynamics is 
contained within the interval model, then the optimal $\mathrm{KF}$ estimate is also contained within the IKF bounds.

The weighted IKF (wIKF) state estimate is then simply a weighted average of the IKF bounds

$$
\begin{aligned}
& \hat{\boldsymbol{x}}^{\mathrm{wIKF}}(k) \underline{\underline{\operatorname{def}}} \min \left(\hat{\boldsymbol{x}}^{\mathrm{IKF}}(k)\right) \\
& +w\left[\max \left(\hat{\boldsymbol{x}}^{\mathrm{IKF}}(k)\right)-\min \left(\hat{\boldsymbol{x}}^{\mathrm{IKF}}(k)\right)\right]
\end{aligned}
$$

where $\hat{\boldsymbol{x}}^{\mathrm{IKF}}(k)$ is the IKF state estimate at time step $k$.

Consider the case $l=1$ (single output system). The IKF estimate of the system output is then the interval value $y^{\mathrm{IKF}}(k)=\mathbf{C}^{\mathrm{I}} \hat{\boldsymbol{x}}^{\mathrm{IKF}}(k)$. A desired weighting sequence $\{w(k)\}$ can be thought of as that which applied to the bounds of $y^{\mathrm{IKF}}(k)$ yields the estimates of an ideal KF (one based on the actual system dynamics) at every time step, that is

$$
\begin{aligned}
& w(k) \in[1,0]: \min \left(\mathbf{C}^{\mathrm{I}} \hat{\boldsymbol{x}}^{\mathrm{KF}}(k)\right) \\
& +w(k)\left[\max \left(\mathbf{C}^{\mathbf{I}} \hat{\boldsymbol{x}}^{\mathrm{IKF}}(k)\right)-\max \left(\mathbf{C}^{\mathbf{I}} \hat{\boldsymbol{x}}^{\mathrm{IKF}}(k)\right)\right] \\
& =\mathbf{C} \hat{\boldsymbol{x}}^{\text {ideal } \mathrm{KF}}(k)
\end{aligned}
$$

Research shows how it is possible to train an artificial neural network (ANN) to model the correlation between the innovations of a nominal KF (based on some nominal model contained within the interval model) and the desired weight, ${ }^{17}$ that is, a mapping $\operatorname{inn}_{K F_{\text {rominal }}}(k) \stackrel{\text { ANN }}{\rightarrow} w(k)$ equation (30) is satisfied.

The training of this ANN involves a target, and it is necessary, therefore, to have the ideal KF estimate, hence the correct model of the system. However, the training can be based on simulation alone using hypothesised true and nominal dynamics. It can be shown that the trained ANN can be used to predict the desired weight independently of these hypothetic systems used to train the ANN, as long as they lie within the interval model that describes the bounded uncertainty.

For this particular study, consider the gyroscope susceptible to developing some bias, as low-cost MEMS gyros are typically subject to null drift due to various reasons. ${ }^{18}$ Its reading can then be considered to be the sum of the actual turning rate, a bias, and a measurement noise (equation (31), Figure 8)

$$
\Omega_{0}=\Omega_{i}+b+\omega
$$

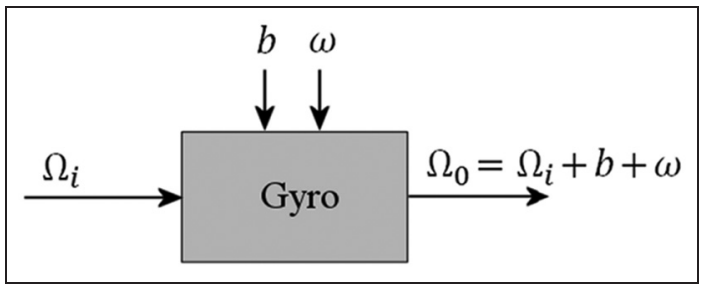

Figure 8. Gyro measurement model: $\Omega_{i}$ is the actual rate of change of heading angle of the vehicle, whereas $\Omega_{0}$ is the value output by the gyroscope mounted on the vehicle.
The predictive model of the vehicle heading based on gyroscopic readings then becomes

$$
\theta(k+1)=\theta(k)+T_{s} \times\left[\Omega_{0}(k)-b-\omega(k)\right]
$$

If the precise value of the gyroscope bias cannot be known (e.g. if it is susceptible to change), but, however, its value can be bounded, $b_{\min }^{\text {gyro }} \leqslant b^{\text {gyro }} \leqslant b_{\max }^{\text {gyro }}$, then this predictive model can be written as an interval model. For the purpose of applying an IKF, the state dynamic and measurement equations may be expressed as

$$
\begin{aligned}
& {\left[\begin{array}{c}
x(k+1) \\
0
\end{array}\right]=\left[\begin{array}{ll}
1 & 0 \\
0 & 0
\end{array}\right]\left[\begin{array}{c}
x(k) \\
0
\end{array}\right]} \\
& +\left[\begin{array}{cc}
T_{s} & -T_{s}\left[b_{\text {min }}^{\text {gyro }}, b_{\text {max }}^{\text {gyro }}\right.
\end{array}\right]\left[\begin{array}{c}
u(k) \\
0 \\
0
\end{array}\right]+\left[\begin{array}{c}
-T_{s} \omega(k) \\
0
\end{array}\right] \\
& z_{\theta}(k)=\left[\begin{array}{ll}
1 & 0
\end{array}\right]\left[\begin{array}{c}
x(k) \\
0
\end{array}\right]+\nu(k)
\end{aligned}
$$

where $x(k)$ represents the vehicle heading, $\theta(k)$. The IKF then yields an interval estimate at each time step, $\hat{x}^{\mathrm{IKF}}(k)$. A point-valued model contained in the interval model (33) would simply be

$$
\begin{aligned}
& {\left[\begin{array}{c}
x(k+1) \\
0
\end{array}\right]=\left[\begin{array}{ll}
1 & 0 \\
0 & 0
\end{array}\right]\left[\begin{array}{c}
x(k) \\
0
\end{array}\right]} \\
& +\left[\begin{array}{cc}
T_{s} & -T_{s} b^{g y r o} \\
0 & 0
\end{array}\right]\left[\begin{array}{c}
u(k) \\
1
\end{array}\right]+\left[\begin{array}{c}
-T_{s} \omega(k) \\
0
\end{array}\right] \\
& z_{\theta}(k)=\left[\begin{array}{ll}
1 & 0
\end{array}\right]\left[\begin{array}{c}
x(k) \\
0
\end{array}\right]+\nu(k)
\end{aligned}
$$

for some value $b^{\text {gyro }} \in\left[b_{\min }^{\text {gyro }}, b_{\text {max }}^{\text {gyro }}\right]$.

Consider an example in which $b_{\min }^{\text {gyro }}=-4^{\circ} \mathrm{s}^{-1}$ and $b_{\text {max }}^{\text {gyro }}=4^{\circ} \mathrm{s}^{-1}$. In order to train an ANN as described earlier, a training mission was established consisting of a different set of way-points. In it, an IKF was simulated along with a KF based on a nominal model given by equations (35) and (36) with $b^{\text {gyro }}=0$. The gyroscope readings were simulated for different lengths of time with different biases between -4 and $4^{\circ} \mathrm{s}^{-1}$, and an ideal KF was also simulated (based on a model given by equations (35) and (36) with $b^{\text {gyro }}$ always being equal to the 'true' bias or bias used to simulate the gyroscope readings). The ANN trained was a feedforward multi-layer perceptron with three time delays in the inputs, one hidden layer of five neurons with hyperbolic tangent activation functions and a linear output neuron.

Figure 9 depicts a simulation of the original waypoint mission described in section 'Introduction and background'. The gyroscope readings were simulated with biases of $-1^{\circ} \mathrm{s}^{-1}$ for $0 \leqslant k \leqslant 300,3.95^{\circ} \mathrm{s}^{-1}$ for $300<k \leqslant 650$ and of $-3.75^{\circ} \mathrm{s}^{-1}$ for the remainder of the simulation (Figure 9(a) and (b)). The heading of the vehicle is shown in Figure 9(c) along with the interval estimates from an IKF. Figure 9(d) shows the nominal KF estimates and those of a wIKF using the previously trained ANN. It clearly shows that while the KF 


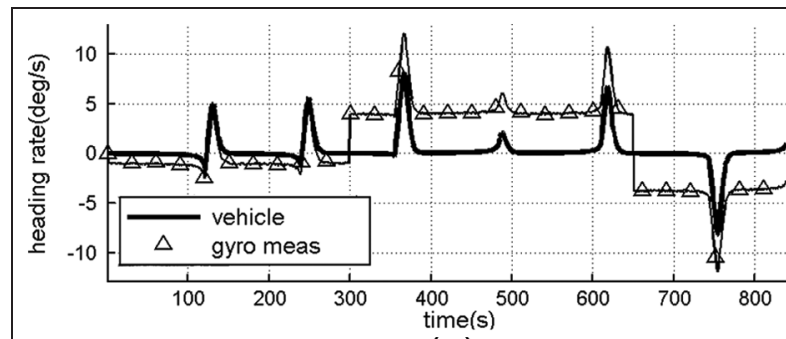

(a)

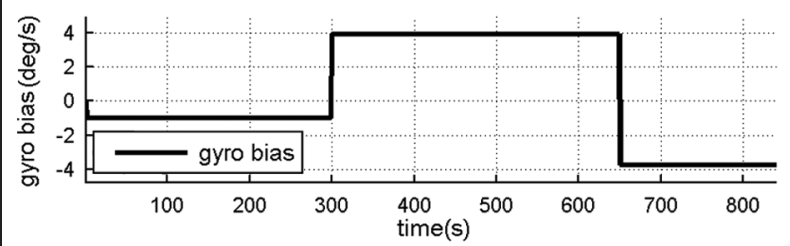

(b)

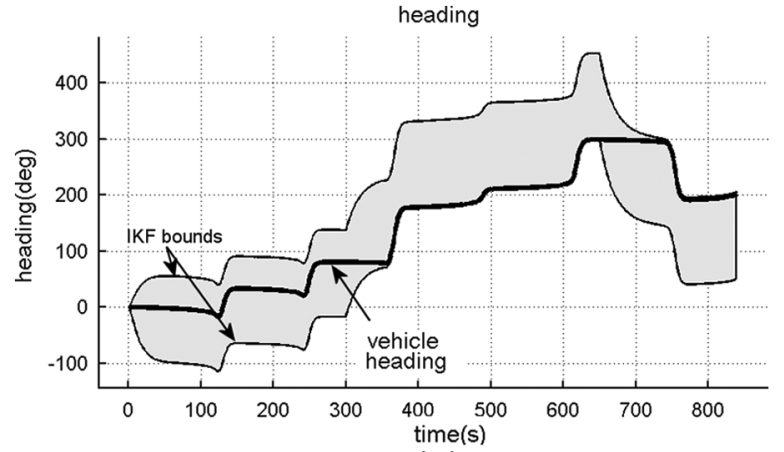

(c)

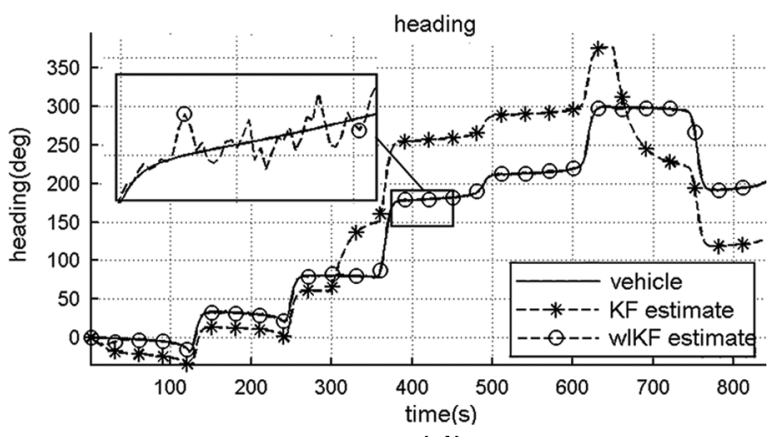

(d)

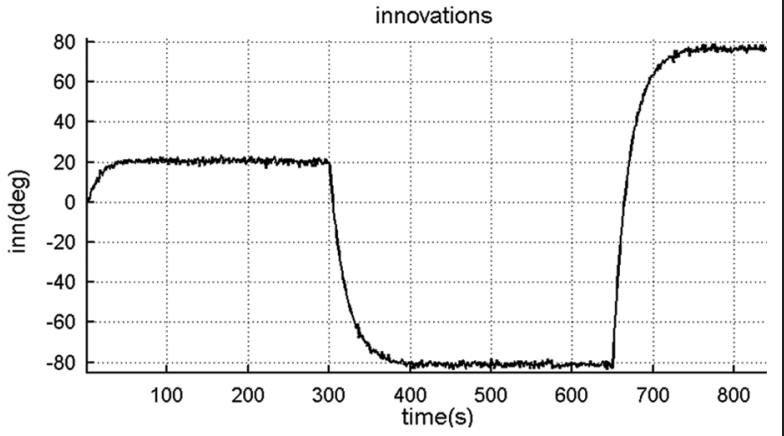

(e)

Figure 9. Simulation of way-point tracking mission with imposed gyroscope bias: (a) actual turning rate and gyroscope measurement; (b) gyroscope bias; (c) actual heading and IKF estimate bounds; (d) actual heading, nominal KF estimate and wIKF estimate; and (e) nominal KF innovations.

IKF: interval Kalman filter; KF: Kalman filter; wIKF: weighted interval Kalman filter.

estimate is degraded due to the incorrect model used, the wIKF provides accurate estimates. Finally, Figure 9(e) shows the innovation sequence of the nominal $\mathrm{KF}$, which is no longer white.

As a note regarding stability considerations, it should be stated that while the KFs constructed upon equations (35) and (36) are easily seen to be stable, the stability of the IKF constructed upon the containing interval model (equations (33) and (34)) does not immediately follow. The reason is not to do with the filter equations themselves but with their implementation. Execution of the algorithm requires operation with interval quantities using interval arithmetic (IA). The properties of IA are defined ${ }^{19}$ in such a way that calculations on interval elements yield further interval elements which guarantee to enclose (although not necessarily be equal to) the actual solution set, a fundamental quality known as the inclusion property, and by virtue of which the IKF bounds guarantee to contain the estimates of every KF based on a model contained within the interval model. However, this conservatism of IA can result in IKF bounds that diverge, even when the actual solution set remains bounded. It is to be stressed that the problem is one of the implementation of the IA calculations and not with the filter equations per se. If IA could be carried out with infinite sharpness, then the IKF would yield stable bounds if the containing KFs were all stable. However, this is not the case in practice, and the propensity of the IKF bounds to diverge depends on the underlying system dynamics. A detailed analysis is beyond the scope of this article, but techniques to improve the tightness of the IKF bounds have been the object of recent study ${ }^{20,21}$ and have been used in implementing the IKF used herein.

\section{Robust and fault-tolerant heading estimation}

Here, fault tolerance refers to being able to operate despite compass failure, a quality which the fuzzy KF fusion algorithm was designed to provide by exploiting the sensor redundancy. However, robustness is used with reference to a KF being able to predict accurate heading estimates even in the face of modelling uncertainty, in this case, unknown (but bounded) gyroscope bias, through the use of the wIKF. This section proposes the fuzzy fusion of wIKF estimates to provide both fault tolerance and robustness. 


\section{Fusion of WIKF estimates and simulation results}

Consider the same way-point tracking simulation of section 'Fuzzy data fusion algorithm for Kalman estimates', with estimates of three KFs associated with three compasses (Table 3), and during which the readings of the compasses associated with $\mathrm{KF}_{3}$ and $\mathrm{KF}_{1}$ are frozen as before at $k=150$ and $k=350$, respectively. In addition, however, the same gyroscope biases described in section 'Robustness and interval Kalman filtering' are prescribed, while the KFs assume zero gyroscope bias models.

The simulation results are shown in Figure 10, with the true heading and three KF estimates shown in Figure 10(a), along with the fused estimate of the same. Because none of the KF estimates are accurate, neither is the fused estimate. Moreover, inspection of the KF innovations (Figure 10(b)) reveals that these are not white even during the period before compass failure occurs. Hence, even the assignment of weights by the fuzzy algorithm is unsatisfactory, as seen in Figure $10(\mathrm{~d})$, where $\mathrm{KF}_{3}$ is eventually awarded the largest weight, even though its estimate is completely erroneous.

The question that remains is whether the fuzzy logic-based algorithm would work to fuse the wIKF estimates, which are, as shown in the previous section, robust to gyroscope biases. However, no standard definition of the wIKF innovation sequence currently exists. The most intuitive proposition would be to define the wIKF innovations as weighted averages of the corresponding IKF innovations (which are intervals at each time step), applying the same weights used to compute the wIKF state and output estimates. It turns out, however, that the most adequate weights for defining the wIKF innovations are instead the complements to unity of these.

Conjecture. Let $\hat{\boldsymbol{x}}^{\mathrm{IKF}}(k)$ be the IKF state estimate of a system based on an interval state-space model as given by equations (26) and (27) and $\hat{\boldsymbol{x}}^{\mathrm{KF}}(k)$ the estimate of a $\mathrm{KF}$ based on some point-valued model contained within the interval model. Consider the weights

$$
\begin{aligned}
& w(k) \in[1,0]: \min \left(\mathbf{C}^{\mathbf{I}} \hat{\boldsymbol{x}}^{\mathrm{IKF}}(k)\right) \\
& +w(k)\left[\max \left(\mathbf{C}^{\mathrm{I}} \hat{\boldsymbol{x}}^{\mathrm{IKF}}(k)\right)-\min \left(\mathbf{C}^{\mathbf{I}} \hat{\boldsymbol{x}}^{\mathrm{IKF}}(k)\right)\right] \\
& =\mathbf{C} \hat{\boldsymbol{x}}^{\mathrm{KF}}(k)
\end{aligned}
$$

and

$$
\begin{aligned}
& w_{2}(k): z(k)-\left\{\min \left(\mathbf{C}^{\mathbf{I}} \hat{\boldsymbol{x}}^{\mathrm{IKF}}(k \mid k-1)\right)\right. \\
& +w_{2}(k)\left[\max \left(\mathbf{C}^{\mathrm{I}} \hat{\boldsymbol{x}}^{\mathrm{IKF}}(k \mid k-1)\right)\right. \\
& \left.\left.-\min \left(\mathbf{C}^{\mathbf{I}} \hat{\boldsymbol{x}}^{\mathrm{IKF}}(k \mid k-1)\right)\right]\right\}=z(k)-\mathbf{C} \hat{\boldsymbol{x}}^{\mathrm{KF}}(k \mid k-1)
\end{aligned}
$$

where $z(k) \in \Re$ is the measurement at time step $k$, and $\hat{\boldsymbol{x}}^{\mathrm{IKF}}(k \mid k-1)$ and $\hat{\boldsymbol{x}}^{\mathrm{KF}}(k \mid k-1)$ are the predictions by the IKF and KF, respectively, of the system state at

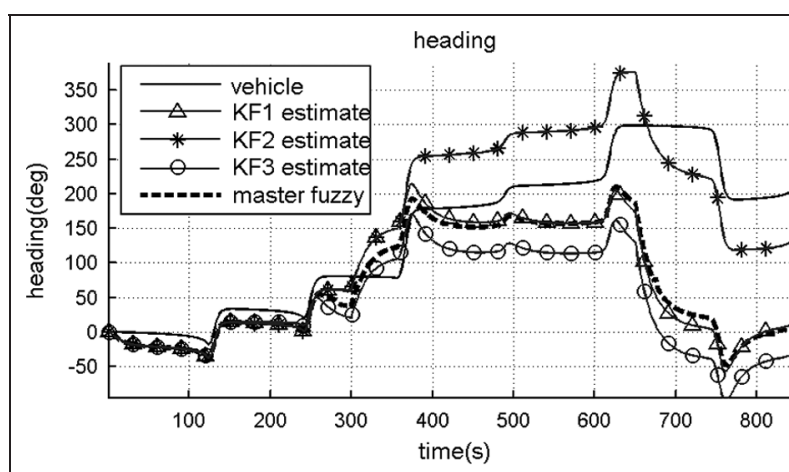

(a)

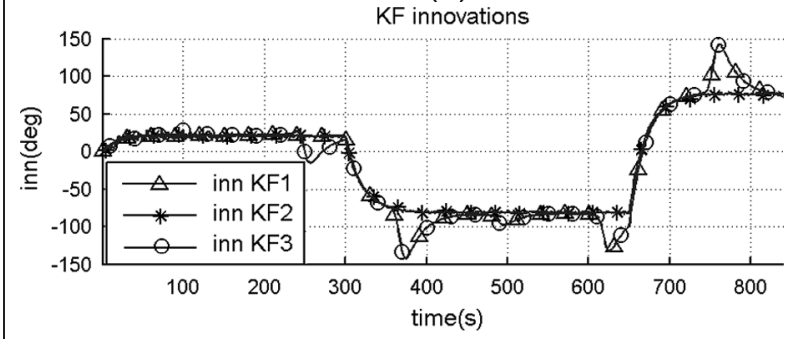

(b)

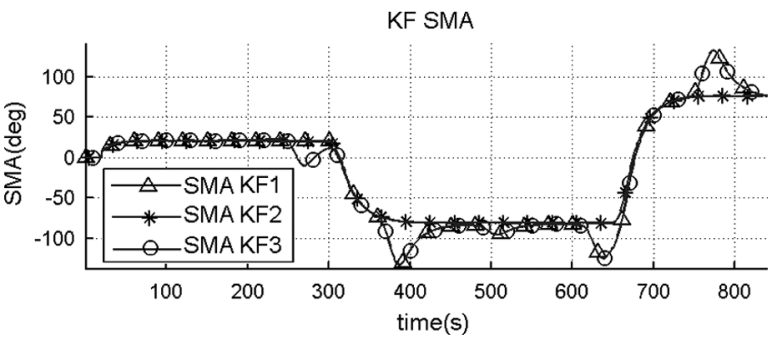

(c)

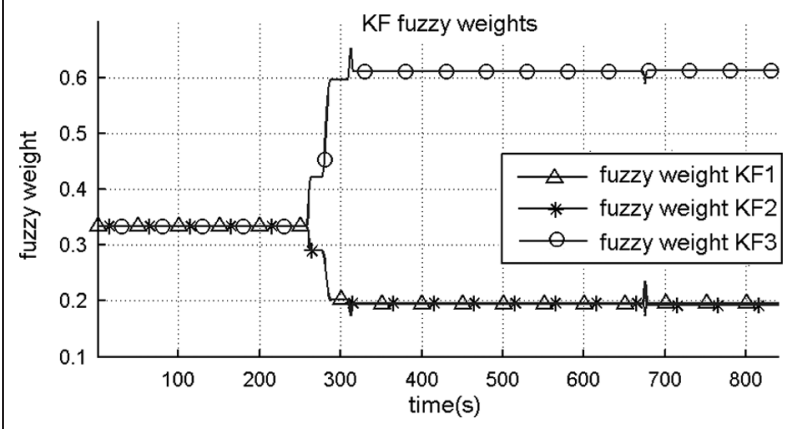

(d)

Figure 10. Simulation of way-point tracking mission with imposed gyroscope bias: (a) actual heading, KF estimates and fused KF estimates; (b) KF innovations; (c) SMA of KF innovations; and (d) fuzzy weights assigned to each KF. KF: Kalman filter; SMA: simple moving average.

time step $k$ given measurements up to time step $k-1$. Then, $w_{2}(k) \approx 1-w(k)$.

Based upon this conjecture, the innovations of the wIKF calculated as

$$
\begin{aligned}
& z(k)-\left\{\min \left(\mathbf{C}^{\mathrm{I}} \hat{\boldsymbol{x}}^{\mathrm{IKF}}(k \mid k-1)\right)\right. \\
& +(1-w(k))\left[\max \left(\mathbf{C}^{\mathrm{I}} \hat{\boldsymbol{x}}^{\mathrm{IKF}}(k \mid k-1)\right)\right. \\
& \left.\left.-\min \left(\mathbf{C}^{\mathrm{I}} \hat{\boldsymbol{x}}^{\mathrm{IKF}}(k \mid k-1)\right)\right]\right\}
\end{aligned}
$$


Table 6. Heading RMS errors.

\begin{tabular}{ll}
\hline Method & Heading RMS error $\left(^{\circ}\right)$ \\
\hline $\mathrm{KF}_{1}$ & 120.37 \\
$\mathrm{KF}_{2}$ & 57.55 \\
$\mathrm{KF}_{3}$ & 145.92 \\
Fused KF estimate & 115.64 \\
wIKF $_{1}$ & 115.36 \\
wIKF $_{2}$ & 1.54 \\
wIKF $_{3}$ & 147.78 \\
Fused wIKF estimate & 6.49 \\
\hline
\end{tabular}

RMS: root mean square; wIKF: weighted interval Kalman filter.

with $w(k)$ obtained as the ANN prediction of the weight in equation (30), approximates an innovation of an ideal KF, through which the fuzzy fusion algorithm may be applied to fuse wIKF estimates.

For the previously described simulated way-point tracking mission, three wIKFs, constructed as described in section 'Robustness and interval Kalman filtering', were simulated to combine gyroscope and compass data, using the same values of $b_{\text {min }}^{\text {gyro }}$ and $b_{\text {max }}^{\text {gyro }}$, and the same trained ANN used therein. Each wIKF is associated with a single compass and initialised with $\hat{x}_{i}^{\mathrm{WIKF}}(0)=\theta(0), \quad \operatorname{var}\left(x-\hat{x}_{i}^{w I K F}(0)\right)=0 . \quad$ Figure 11 depicts the simulation results: Figure 11(a) compares the actual vehicle's heading to those obtained from each wIKF, as well as the fused wIKF estimate. The innovations of each wIKF, and the SMA of these, are shown in Figure 11(b) and (c), respectively. It can be seen how the innovations, defined as in equation (39), are mostly composed of white noise sequences prior to compass failure, except for small transient periods after a sudden change in gyroscope bias, as the ANN weight prediction requires time to adapt to the new dynamics. However, these transients are common to all three wIKFs, and so, none of them are discriminated during the same. However, after compass failure, the innovations deviate substantially from the ideal, especially during sharp turning manoeuvres, and it is these deviations that result in the fusion algorithm penalising the corresponding wIKF weights, as shown in Figure 11(d).

The RMS heading errors of the KFs and fused KF estimate shown in Figure 10, together with those of the wIKFs and fused wIKF estimate of Figure 11, are given in Table 6. Both figures and table evince the robust and fault-tolerant qualities of the fused wIKF estimate in the face of sporadic compass failure and gyroscope bias drifts.

\section{Real-time trial results}

Trials to test the proposed fault-tolerant navigation system were conducted with Springer at Roadford Lake in Devon, United Kingdom, on 2 July 2014, using the units described in Table 1. The mission, consisting of four way-points (three buoys), is shown in Figure 12.

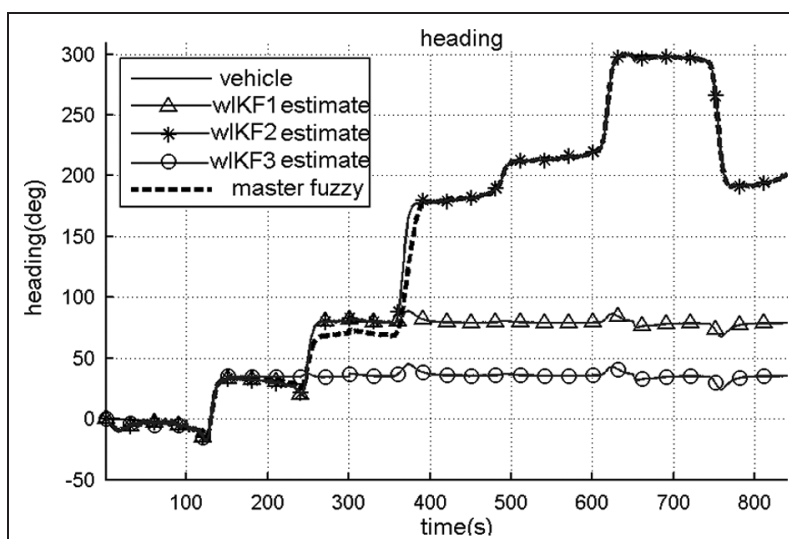

(a)

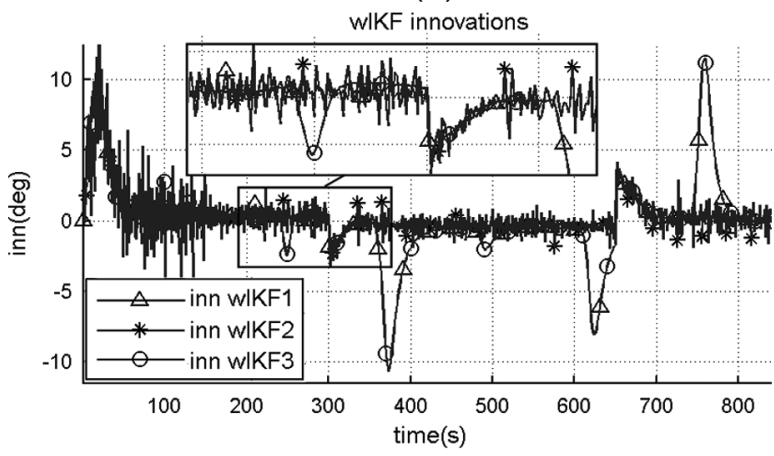

(b)

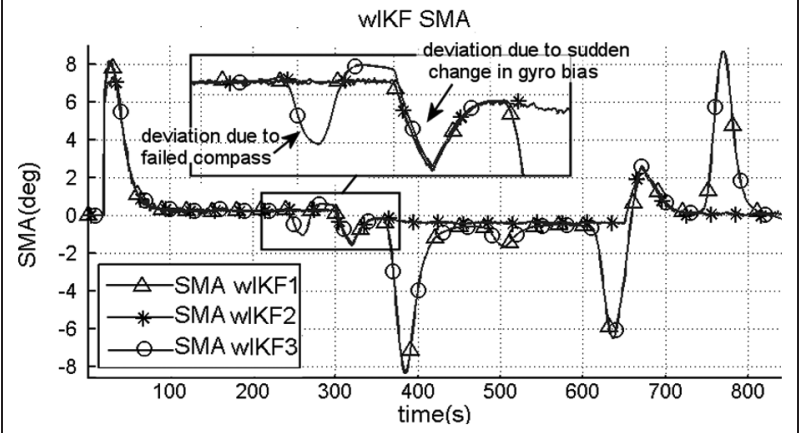

(c)

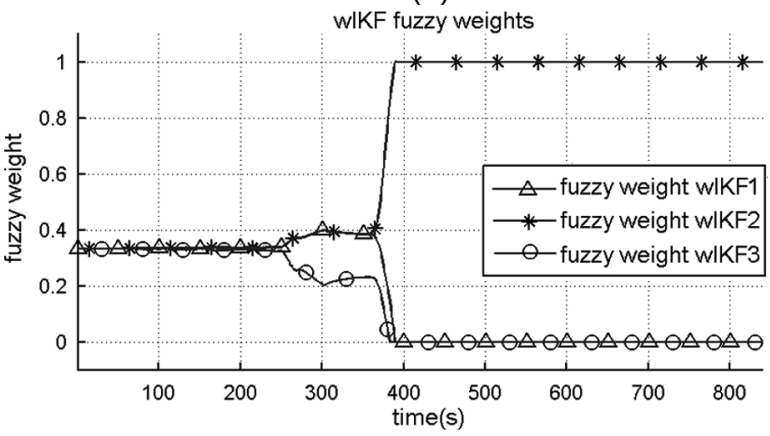

(d)

Figure I I. Simulation of way-point tracking mission with imposed gyroscope bias: (a) actual heading, wIKF estimates and fused wIKF estimates; (b) wIKF innovations; (c) SMA of wIKF innovations; and (d) fuzzy weights assigned to each wIKF. wIKF: weighted interval Kalman filter; SMA: simple moving average.

The GPS coordinates of these buoys were obtained prior to the trials and programmed into the vehicle's guidance system. The starting point of the mission was located at a jetty from where the vehicle could be 


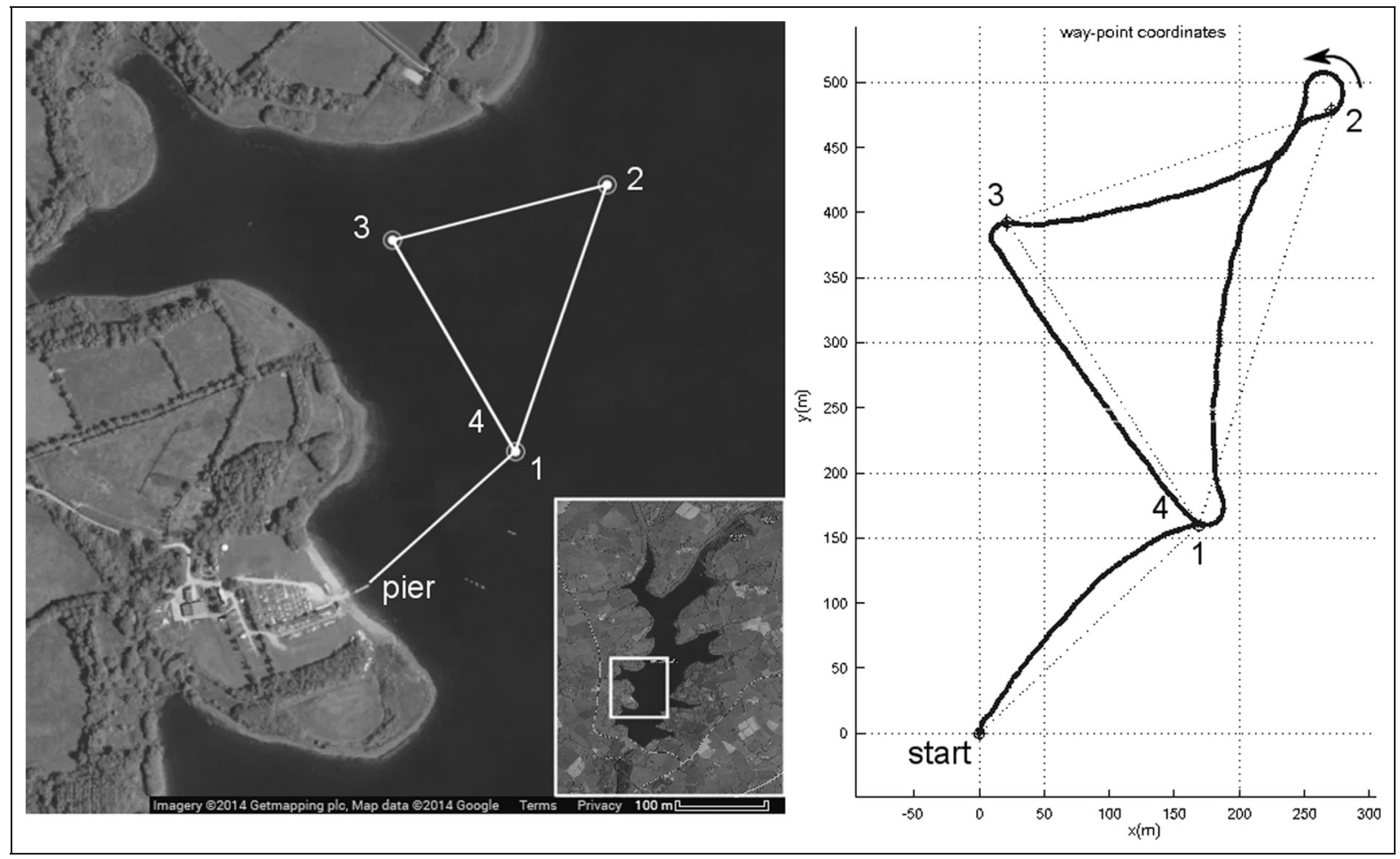

Figure I2. Way-point mission established at Roadford Lake and actual vehicle trajectory during trials.

launched. Figure 12 also depicts the actual trajectory taken by the vehicle during one of the trials, which is described next.

The data shown in Figure 13 correspond to a trial in which two of the compasses were made to fail. The gyroscope readings are shown in Figure 13(a), with the gyroscope having a bias of roughly $-3.5 \mathrm{~m} \mathrm{~s}^{-1}$. The compass readings are shown in Figure 13(b). The readings of the TCM2 were frozen at $k=182 \mathrm{~s}$, roughly halfway to the first way-point, whereas those of the HMR3000 were frozen at $k=472 \mathrm{~s}$, approximately $50 \mathrm{~m}$ before reaching the second. The individual gyrocompass wIKF heading estimates, labelled 1-3 for TCM2, HMR300 and KVH C100, respectively, are shown in Figure 13(c), along with the fused wIKF. For completeness, the innovations of each wIKF, SMA values and fuzzy weights are shown in Figure 13(d)-(f), respectively. The heading estimates used by the autopilot during the trial were those of the fused wIKF. Wind speed was also monitored during the trial and averaged from 1 to $2 \mathrm{~m} \mathrm{~s}^{-1}$ in a north-westerly direction, with gusts of up to $5 \mathrm{~m} \mathrm{~s}^{-1}$.

Table 7 benchmarks the various wIKF estimates and fused wIKF against the wIKF corresponding to the $\mathrm{KVH} \mathrm{C100} \mathrm{compass} \mathrm{(} \mathrm{wIKF}_{3}$ ), as this was the only compass that was not made to fail. It should be noted that the error of the fused wIKF was transient, occurring during the periods following the respective compass failures but recovering in due course (Figure 13(c)), while the errors shown in Table 7 are averaged values for the duration of the mission; thus, the fused wIKF RMS error in particular would, therefore, tend to zero
Table 7. Roadford trial results.

\begin{tabular}{ll}
\hline Method & Heading RMS error $\left(^{\circ}\right)$ \\
\hline wIKF $_{1}($ gyro - TCM2) & 160.4 \\
WIKF $_{2}$ (gyro - HMR 3000) & 156.1 \\
wIKF $_{3}$ (gyro - KVH Cl00) & 0.0 \\
Fused wIKFs & 16.5 \\
\hline
\end{tabular}

RMS: root mean square; wIKF: weighted interval Kalman filter.

as the mission length increased. The mission was repeated four times, with compass failures provoked at different instances, yielding similar results. A similar mission was also attempted with the autopilot using feedback from fused ordinary KF estimates, but even without compass failure, the vehicle was unable to complete the mission. Thus, the trial results corroborate the importance of an accurate heading estimation subsystem for the vehicle to operate successfully, and that the method proposed in this article achieves this, demonstrating its robustness to unaccounted-for shifts in gyroscope bias as well as sporadic failure of up to two of the three available compasses.

\section{Concluding statements}

The research presented in this article concerns the design of a MSDF algorithm for fusing data from various KFs associated with different compass units in order to detect compass failure and penalise the corresponding KF's contribution to the fused estimate. Although only one type of compass failure was shown 


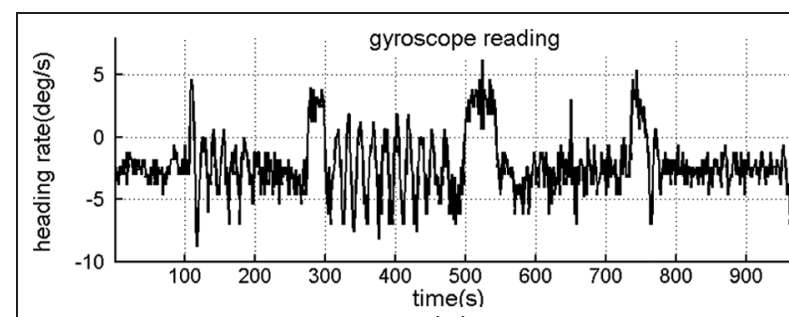

(a)

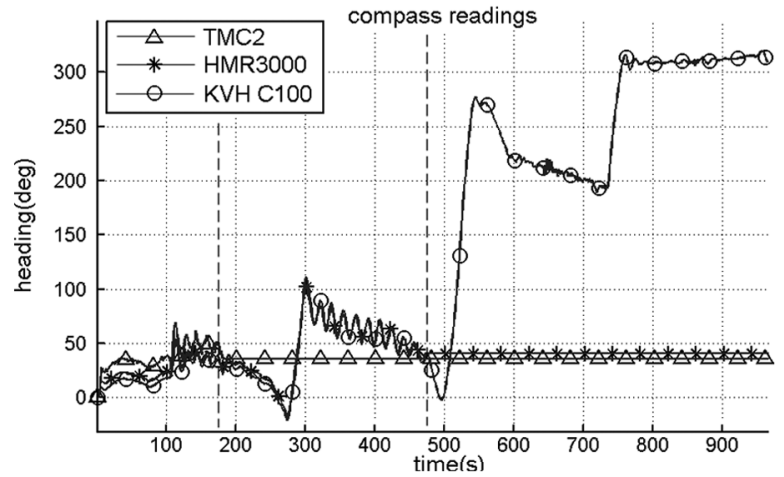

(b)

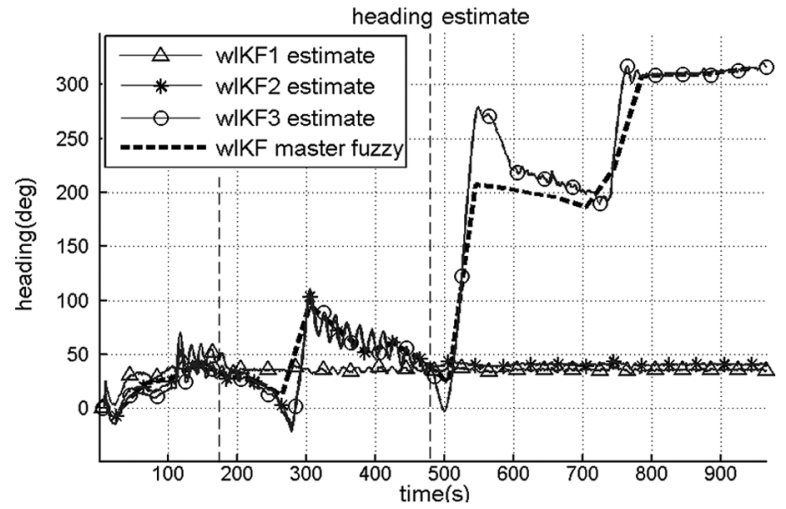

(c)

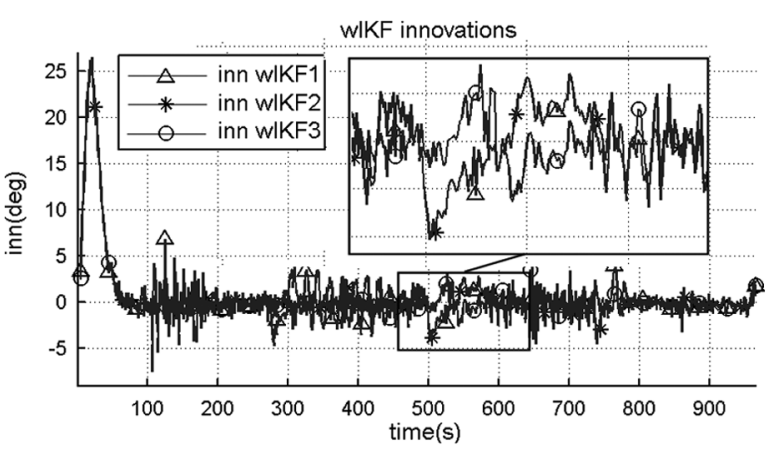

(d)

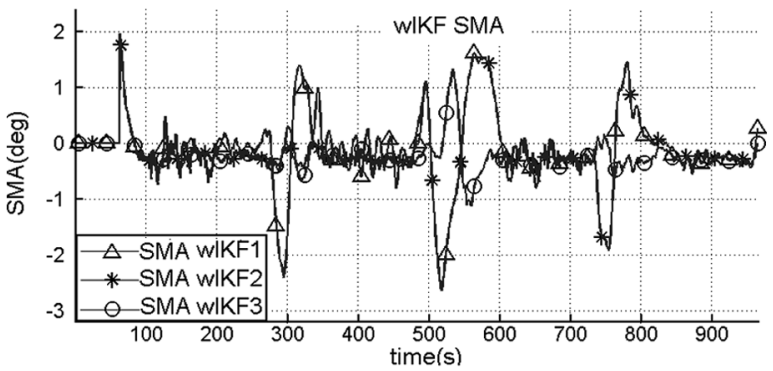

(e)

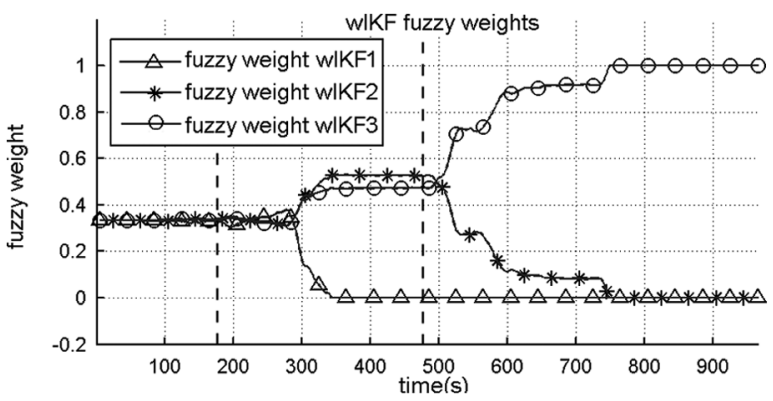

(f)

Figure 13. Roadford trials results: (a) gyroscope measurements, (b) compass measurements, (c) wIKF estimates and fused wIKF estimates, (d) wIKF innovations, (e) SMA of each wIKF and (f) fuzzy weights assigned to each wIKF. wIKF: weighted interval Kalman filter; SMA: simple moving average.

in which the reading remained frozen after a certain period of time, this is, in fact, quite a subtle kind of fault to detect - the algorithm would work equally well (or better) to detect faults such as a permanent zero compass reading. Application of this algorithm in realtime trials allowed the vehicle to continue successful autonomous operation even when all but one of its compass units failed to provide correct readings. Such capability is referred to as fault tolerance.

In order to provide a degree of robustness to system modelling uncertainty, in this case caused by a drifting gyroscope bias, the wIKF was proposed as a solution. When the innovations of the wIKF are appropriately defined, the fault-tolerant fusion algorithm can be applied to fuse wIKF estimates. This confers the heading estimation subsystem with both robustness and fault tolerance. The importance of both these qualities was demonstrated in real-time trials, allowing the vehicle to successfully complete its mission, while showing that the method can be effectively implemented in practice.

While this article has focused its application to the particular navigation problem of heading estimation, the algorithm developed can easily be extended to more or even other types of sensors and applied in general where KFs apply and sensor redundancy exists. For example, Kalman filtering is widely used to fuse GPS and inertial data for position estimates, and often more than one GPS receiver or accelerometer units are available to prevent loss of information. Furthermore, since the MSDF technique developed here has shown that the same basic fusion architecture applied to wIKFs rather than KFs can enhance the reliability of the estimate by assuming the inherent robustness of the wIKF, it is suggestive of the potential benefits that research into the extension of other established MSDF techniques that employ KFs to work with wIKFs could bring. 


\section{Declaration of conflicting interests}

The authors declared no potential conflicts of interest with respect to the research, authorship and/or publication of this article.

\section{Funding}

This work was supported by the Engineering and Physical Sciences Research Council (grant number EP/ I012923/1).

\section{References}

1. Motwani A. Survey of uninhabited surface vehicles. Technical report MIDAS.SMSE.2012.TR.001, 22 April 2012. Plymouth: Plymouth University.

2. Bijker J and Steyn W. Kalman filter configurations for a low-cost loosely integrated inertial navigation system on an airship. Control Eng Pract 2008; 16(12): 1509-1518.

3. Rodriguez R and Gomez J. Analysis of three different Kalman filter implementations for agricultural vehicle positioning. Open Agr J 2009; 3: 13-19.

4. Zhang P, Gu J, Milios EE, et al. Navigation with IMU/ GPS/Digital compass with unscented Kalman filter. In: Proceedings of IEEE, international conference on mechatronics and automation, Niagara Falls, ON, Canada, July 2005. New York: IEEE.

5. Zhao $\mathrm{H}$ and Wang Z. Motion measurement using inertial sensors, ultrasonic sensors, and magnetometers with extended Kalman filter for data fusion. IEEE Sens $J$ 2012; 12(5):943-953.

6. Xu T. An intelligent navigation system for an unmanned surface vehicle. PhD Thesis, Plymouth University, Plymouth, 2007.

7. Naeem $\mathrm{W}, \mathrm{Xu} T$, Sutton R, et al. The design of a navigation guidance and control system for an unmanned surface vehicle for environmental monitoring. Proc IMechE, Part M: J Engineering for the Maritime Environment 2008; 222(2): 67-79.

8. TinkerKit. 4x gyroscope manual, http://www.tinkerkit. com/gyroscope-4x/ (2014, accessed 10 April 2014).

9. PNI. Compass TCM2 manual, http://www.mil.ufl.edu/ projects/gnuman/gnuman_pre2005/spec_sheets/tcm2_ productsheet.pdf (2014, accessed 10 April 2014).

10. Honeywell. Compass HMR3000 manual, http://web.ar rownac.com/sites/default/files/pdfs/HMR3000.pdf (2014, accessed 10 April 2014).

11. KVH. Compass KVH C100 manual, http://www.canal geomatics.com/product_files/kvh-c100-compass_426.pdf (2014, accessed 10 April 2014).

12. Annamalai ASK, Sutton R, Yang C, et al. Robust adaptive control of an uninhabited surface vehicle. $J$ Intell Robot Syst 2014; 78(2): 319-338.

13. Chui CK and Chen G. Kalman filtering with real-time applications. 4th ed. New York: Springer, 2008.

14. Lewis F, Xie L and Dopa D. Optimal and robust estimation: with an introduction to stochastic control theory. 2nd ed. Boca Raton, FL: CRC Press, 2007.

15. Subramanian V, Burks TF and Dixon WE. Sensor fusion using fuzzy logic enhanced Kalman filter for autonomous vehicle guidance in citrus groves. T ASABE 2009; 52(5): $1411-1422$.
16. Chen G, Wang J and Shieh LS. Interval Kalman filtering. IEEE T Aero Elec Sys 1997; 33(1): 250-258.

17. Motwani A, Sharma S, Sutton R, et al. Application of artificial neural networks to weighted interval Kalman filtering. Proc IMechE, Part I: J Systems and Control Engineering 2014; 228(5): 267-277.

18. Shiau JK, Huang CX and Chang MY. Noise characteristics of MEMS Gyro's null drift and temperature compensation. J Appl Sci Eng 2012; 15(3): 239-246.

19. Moore RE. Interval analysis. Englewood, NJ: Prentice Hall, 1966.

20. Motwani A, Sharma S, Sutton R, et al. On the application of a hybrid ellipsoidal-rectangular interval arithmetic algorithm to interval Kalman filtering for state estimation of uncertain systems. Int J Control 2015; 88(9): 1805-1817.

21. Motwani A, Sharma S, Sutton R, et al. Computation of stable interval Kalman filter bounds for their use in robust state estimation for an uninhabited surface vehicle with bounded indeterminate system dynamics. In: Proceedings of the IEEE intelligent vehicles symposium, Dearborn, MI, 8-11 June 2014, pp.356-361. New York: IEEE.

\section{Appendix I}

\section{Vehicle simulation and autopilot}

\section{Vehicle model}

During system identification trials at Roadford Lake, the vehicle was made to undergo various steering manoeuvres at constant speed. The differential propeller speed input and heading output were recorded, and the following discrete state-space model for a constant vehicle speed of $1.5 \mathrm{~m} \mathrm{~s}^{-1}$ was obtained

$$
\begin{aligned}
& \boldsymbol{x}(k+1)=\mathbf{A} \boldsymbol{x}(k)+\mathbf{B} u(k) \\
& y(k)=\mathbf{C} \boldsymbol{x}(k)
\end{aligned}
$$

with

$$
\begin{aligned}
& \mathbf{A}=\left[\begin{array}{cc}
0.4 & -0.1025 \\
1 & 0
\end{array}\right], \mathbf{B}=\left[\begin{array}{l}
1 \\
0
\end{array}\right], \\
& \mathbf{C}=\left[\begin{array}{ll}
0 & 0.007025
\end{array}\right] \text { and } T_{s}=1 \mathrm{~s}
\end{aligned}
$$

between the differential propeller speed input $u(k)$ (in $\mathrm{r} \mathrm{m}^{-1}$ ) and the rate of change of the heading angle, $y(k)\left(\right.$ in $\left.{ }^{\circ} \mathrm{s}^{-1}\right)$. The heading of the vehicle is then simply obtained as

$$
\theta(k+1)=\theta(k)+T_{s} y(k)=\theta(k)+T_{s} \mathbf{C} \boldsymbol{x}(k)
$$

and the position of the vehicle updated according to

$$
\begin{aligned}
& \left(x^{\mathrm{ASV}}(k), y^{\mathrm{ASV}}(k)\right)=\left(x^{\mathrm{ASV}}(k-1), y^{\mathrm{ASV}}(k-1)\right) \\
& +T_{s} v\left(\cos \left(\frac{\pi}{180} \frac{\theta(k+1)+\theta(k)}{2}\right)\right. \\
& \left.\sin \left(\frac{\pi}{180} \frac{\theta(k+1)+\theta(k)}{2}\right)\right)+T_{S}(0,0.1)
\end{aligned}
$$

with $T_{s}=1 \mathrm{~s}$ and $v=1.5 \mathrm{~m} \mathrm{~s}^{-1}$. 


\section{Autopilot}

With the heading as output, the vehicle steering model can be written as

$$
\begin{aligned}
& {\left[\begin{array}{c}
x_{1}(k+1) \\
x_{2}(k+1) \\
\theta(k+1)
\end{array}\right]=\left[\begin{array}{c:c}
\mathbf{A} & 0 \\
\hdashline \mathbf{C} & 1
\end{array}\right]\left[\begin{array}{c}
x_{1}(k) \\
x_{2}(k) \\
\theta(k)
\end{array}\right]+\left[\begin{array}{c}
\mathbf{B} \\
\hdashline 0
\end{array}\right] u(k)} \\
& \theta(k)=\left[\begin{array}{lll}
0 & 0 & 1
\end{array}\right]\left[\begin{array}{c}
x_{1}(k) \\
x_{2}(k) \\
\theta(k)
\end{array}\right]
\end{aligned}
$$

or with the appropriate definitions, expressed compactly as

$$
\begin{aligned}
& \tilde{\boldsymbol{x}}(k+1)=\widetilde{\mathbf{A}} \tilde{x}(k)+\widetilde{\mathbf{B}} u(k) \\
& \theta(k)=\widetilde{\mathbf{C}} \tilde{\boldsymbol{x}}(k)
\end{aligned}
$$

A simple state-feedback control law is

$$
u(k)=-\mathbf{K} \tilde{\boldsymbol{x}}(k)+\mathbf{K}_{\mathrm{s}} r(k)
$$

The values of $\mathbf{K}$ are chosen herein so that the closedloop system dynamics (equation (50)) has a rise time of $10 \mathrm{~s}$, deemed sufficient taking into account that physical constraints would constantly lead to actuator saturation if higher feedback gains were used. The corresponding gain values are $\mathbf{K}=\left[\begin{array}{lll}-0.5500 & 0.2150 & 6.1032\end{array}\right]$. $\mathrm{K}_{\mathrm{S}}$ is a scaling gain selected a posteriori to ensure that the steady-state gain of the closed-loop system (given by equations (50) and (48)) is unity

$$
\tilde{\boldsymbol{x}}(k+1)=(\tilde{\mathbf{A}}-\widetilde{\mathbf{B}} \mathbf{K}) \tilde{\boldsymbol{x}}(k)+\widetilde{\mathbf{B}} \mathrm{K}_{\mathrm{s}} r(k)
$$

\section{Appendix 2}

\section{Kalman filtering}

Consider a dynamic system modelled in discrete time via the following stochastic state-space equations

$$
\begin{aligned}
& \boldsymbol{x}(k+1)=\mathbf{A} x(k)+\mathbf{B} u(k)+\boldsymbol{\omega}(k) \\
& \boldsymbol{z}(k)=\mathbf{C} \quad x(k)+\boldsymbol{\nu}(k)
\end{aligned}
$$

where $\boldsymbol{x}(k)$ is the system state at time $k, \boldsymbol{u}(k)$ a controllable system input, $\boldsymbol{\omega}(k)$ a random input disturbance, $z(k)$ a noisy measurement of the system output and $\boldsymbol{\nu}(k)$ the measurement noise. If $\{\boldsymbol{x}(0), \boldsymbol{\omega}(0), \ldots, \boldsymbol{\omega}(k)$, $\boldsymbol{\nu}(0), \ldots, \boldsymbol{\nu}(k)\}$ are mutually independent, then the KF provides a statistically optimal estimate of the state vector at each time step (equations (53)-(57)).

Prediction

$$
\hat{\boldsymbol{x}}(k \mid k-1)=\mathbf{A} \hat{\boldsymbol{x}}(k-1 \mid k-1)+\mathbf{B} \boldsymbol{u}(k-1)
$$

$$
\mathbf{P}(k \mid k-1)=\mathbf{A} \quad \mathbf{P}(k-1 \mid k-1) \mathbf{A}^{T}+\mathbf{Q}
$$

Kalman gain

$$
\mathbf{K}(k)=\mathbf{P}(k \mid k-1) \mathbf{C}^{T}\left\{\mathbf{C} \mathbf{P}(k \mid k-1) \mathbf{C}^{T}+\mathbf{R}\right\}^{-1}
$$

Correction

$$
\begin{aligned}
& \hat{\boldsymbol{x}}(k \mid k)=\hat{x}(k \mid k-1)+\mathbf{K}(k)\{z(k)-\mathbf{C} \hat{\boldsymbol{x}}(k \mid k-1)\} \\
& \mathbf{P}(k \mid k)=\{\mathbf{I}-\mathbf{K}(k) \mathbf{C}\} \mathbf{P}(k \mid k-1)
\end{aligned}
$$

where $\mathbf{Q}=\operatorname{var}(\boldsymbol{\omega})$ and $\mathbf{R}=\operatorname{var}(\boldsymbol{\nu})$. 